\title{
ENGEL-TYPE SUBGROUPS AND LENGTH PARAMETERS OF FINITE GROUPS
}

\author{
E. I. KHUKHRO AND P. SHUMYATSKY
}

\begin{abstract}
Let $g$ be an element of a finite group $G$. For a positive integer $n$, let $E_{n}(g)$ be the subgroup generated by all commutators $[\ldots[[x, g], g], \ldots, g]$ over $x \in G$, where $g$ is repeated $n$ times. By Baer's theorem, if $E_{n}(g)=1$, then $g$ belongs to the Fitting subgroup $F(G)$. We generalize this theorem in terms of certain length parameters of $E_{n}(g)$. For soluble $G$ we prove that if, for some $n$, the Fitting height of $E_{n}(g)$ is equal to $k$, then $g$ belongs to the $(k+1)$ th Fitting subgroup $F_{k+1}(G)$. For nonsoluble $G$ the results are in terms of nonsoluble length and generalized Fitting height. The generalized Fitting height $h^{*}(H)$ of a finite group $H$ is the least number $h$ such that $F_{h}^{*}(H)=H$, where $F_{0}^{*}(H)=1$, and $F_{i+1}^{*}(H)$ is the inverse image of the generalized Fitting subgroup $F^{*}\left(H / F_{i}^{*}(H)\right)$. Let $m$ be the number of prime factors of $|g|$ counting multiplicities. It is proved that if, for some $n$, the generalized Fitting height of $E_{n}(g)$ is equal to $k$, then $g$ belongs to $F_{f(k, m)}^{*}(G)$, where $f(k, m)$ depends only on $k$ and $m$. The nonsoluble length $\lambda(H)$ of a finite group $H$ is defined as the minimum number of nonsoluble factors in a normal series each of whose factors either is soluble or is a direct product of nonabelian simple groups. It is proved that if $\lambda\left(E_{n}(g)\right)=k$, then $g$ belongs to a normal subgroup whose nonsoluble length is bounded in terms of $k$ and $m$. We also state conjectures of stronger results independent of $m$ and show that these conjectures reduce to a certain question about automorphisms of direct products of finite simple groups.
\end{abstract}

\section{INTRODUCTION}

By the well-known theorem of Baer [2, Satz III.6.15], an element $g$ of a finite group $G$ belongs to the Fitting subgroup $F(G)$ if and only if it is a left-Engel element, that is, if $[x, g, g, \ldots, g]=1$ for all $x \in G$, where $g$ is repeated in the commutator sufficiently many times. (Throughout the paper, we use the left-normed simple commutator notation $\left[a_{1}, a_{2}, a_{3}, \ldots, a_{r}\right]=\left[\ldots\left[\left[a_{1}, a_{2}\right], a_{3}\right], \ldots, a_{r}\right]$.) In this paper we generalize this result using the subgroups

$$
E_{n}(g)=\langle[x, \underbrace{g, \ldots, g}_{n}] \mid x \in G\rangle .
$$

Recall that the Fitting series is defined starting from $F_{0}(G)=1$, and then by induction $F_{k+1}(G)$ is the inverse image of $F\left(G / F_{k}(G)\right)$. Our first result is about soluble groups.

Theorem 1.1. Let $g$ be an element of a finite soluble group $G$, and $n$ a positive integer. If the Fitting height of $E_{n}(g)$ is equal to $k$, then $g$ belongs to $F_{k+1}(G)$.

For nonsoluble finite groups we prove similar results in terms of the nonsoluble length and generalized Fitting height of $E_{n}(g)$. We recall the relevant definitions. The generalized

1991 Mathematics Subject Classification. 20D25, $20 \mathrm{D} 45$.

Key words and phrases. Finite groups, nonsoluble length, generalized Fitting height, commutator subgroup.

The first author was supported by the Russian Science Foundation, project no. 14-21-00065, and the second by the Conselho Nacional de Desenvolvimento Científico e Tecnológico (CNPq), Brazil. The first author thanks CNPq-Brazil and the University of Brasilia for support and hospitality that he enjoyed during his visits to Brasilia. 
Fitting subgroup $F^{*}(G)$ is the product of the Fitting subgroup $F(G)$ and all subnormal quasisimple subgroups (here a group is quasisimple if it is equal to its derived subgroup and its quotient by the centre is a non-abelian simple group). Then the generalized Fitting series of $G$ is defined starting from $F_{0}^{*}(G)=1$, and then by induction $F_{i+1}^{*}(G)$ is the inverse image of $F^{*}\left(G / F_{i}^{*}(G)\right)$. The least number $h$ such that $F_{h}^{*}(G)=G$ is naturally defined to be the generalized Fitting height $h^{*}(G)$ of $G$. Clearly, if $G$ is soluble, then $h^{*}(G)=h(G)$ is the ordinary Fitting height of $G$.

Theorem 1.2. Let $m$ and $n$ be positive integers, and let $g$ be an element of a finite group $G$ whose order $|g|$ is equal to the product of $m$ primes counting multiplicities. If the generalized Fitting height of $E_{n}(g)$ is equal to $k$, then $g$ belongs to $F_{j}^{*}(G)$ for some $j \leqslant((k+1) m(m+1)+2)(k+3) / 2$.

This theorem follows from Theorem 1.1 on soluble groups and Theorem 1.3 below concerning another length parameter. Namely, the nonsoluble length $\lambda(G)$ of a finite group $G$ is defined as the minimum number of nonsoluble factors in a normal series each of whose factors either is soluble or is a direct product of nonabelian simple groups. (In particular, the group is soluble if and only if its nonsoluble length is 0.) Bounds for the nonsoluble length or/and generalized Fitting height of a finite group $G$ greatly facilitate using the classification of finite simple groups (and are themselves often obtained by using the classification). Most notably such bounds were used in the reduction of the Restricted Burnside Problem to soluble and nilpotent groups in the Hall-Higman paper [1]. Such bounds also find applications in the study of profinite groups. Examples include Wilson's reduction of the problem of local finiteness of periodic profinite groups to pro- $p$ groups in [4] and our recent paper [3] on similar problems. (Both the Restricted Burnside Problem and the problem of local finiteness of periodic profinite groups were solved by Zelmanov $[5,6,7]$.

Similarly to the generalized Fitting series, we can define terms of the "upper nonsoluble series': let $R_{i}(G)$ be the maximal normal subgroup of $G$ that has nonsoluble length $i$ (so that, in particular, $R_{0}(G)$ is the soluble radical of $G$ ).

Theorem 1.3. Let $m$ and $n$ be positive integers, and let $g$ be an element of a finite group $G$ whose order $|g|$ is equal to the product of $m$ primes counting multiplicities. If the nonsoluble length of $E_{n}(g)$ is equal to $k$, then $g$ belongs to $R_{j}(G)$ for some $j \leqslant$ $(k+1) m(m+1) / 2$.

Note that $E_{n}(g)$ is not a subgroup of the type $[\ldots[[G, g], g], \ldots, g]$ formed by taking successive commutator subgroups, which is subnormal. But $E_{n}(g)$ is not subnormal in general.

Our results on nonsoluble groups depend on the classification of finite simple groups in so far as the validity of the Schreier conjecture on solubility of the group of outer automorphisms of a finite simple group. In Theorems 1.2 and 1.3, nonsoluble length and generalized Fitting height have bounds depending both on the parameters of $E_{n}(g)$ and on the number of prime factors in the order of $g$. We conjecture that stronger results may hold, not depending on the order of $g$. Namely, we conjecture that if, for an element $g$ of a finite group $G$ the nonsoluble length of $E_{n}(g)$ is equal to $k$, then $g$ belongs to $R_{k}(G)$. We also conjecture that if the generalized Fitting height of $E_{n}(g)$ is equal to $k$, then $g$ belongs to $F_{k+1}^{*}(G)$. These conjectures can be reduced to certain questions about automorphisms of direct products of nonabelian simple groups. The questions, though, seem rather difficult and remain open. We discuss them in $\S 7$.

The authors thank Professor Robert M. Guralnick for useful discussions. 


\section{Preliminaries AND Proof of Theorem 1.1}

We notice nice 'radical' properties of the subgroups $F_{i}(G), F_{i}^{*}(G)$, and $R_{i}(G)$, which will be often used without special references. Namely, it follows from the definitions that if $N$ is a normal subgroup of $G$, then

$$
F_{i}(N)=N \cap F_{i}(G), \quad F_{i}^{*}(N)=N \cap F_{i}^{*}(G), \quad R_{i}(N)=N \cap R_{i}(G) .
$$

For the Fitting subgroups we also have the inclusions $F_{i}(G) \cap H \leqslant F_{i}(H)$ for any, not necessarily normal, subgroup $H \leqslant G$. However, similar inclusions do not hold in general for the subgroups $F_{i}^{*}(G)$ and $R_{i}(G)$.

As a consequence we have the following.

Lemma 2.1. Let $N$ be a subnormal subgroup of a finite group $G$. Then

(a) the Fitting height (when $G$ is soluble), the generalized Fitting height, and the nonsoluble length of $N$ do not exceed the corresponding parameters of $G$, and

(b) the Fitting height (when $N$ is soluble), the generalized Fitting height, and the nonsoluble length of the normal closure $\left\langle N^{G}\right\rangle$ are equal to the corresponding parameters of $N$.

It also follows from the definitions that

$$
F_{i}(G / N) \geqslant F_{i}(G) N / N, \quad F_{i}^{*}(G / N) \geqslant F_{i}^{*}(G) N / N, \quad R_{i}(G / N) \geqslant R_{i}(G) N / N
$$

for any normal subgroup $N$ of $G$.

When we consider a group $A$ acting by automorphisms on a group $G$, we regard $A$ as a subgroup of the natural semidirect product $G A$, so that we can form the mutual commutator subgroup $[G, A]$ and use the centralizer notation for the fixed-point subgroup $C_{G}(A)$. Throughout the paper we use without special references the well-known properties of coprime actions: if $\alpha$ is an automorphism of a finite group $G$ of coprime order, $(|\alpha|,|G|)=1$, then $C_{G / N}(\alpha)=C_{G}(\alpha) N / N$ for any $\alpha$-invariant normal subgroup $N$, the equality $[G, \alpha]=[[G, \alpha],, \alpha]$ holds, and if $G$ is in addition abelian, then $G=[G, \alpha] \times C_{G}(\alpha)$.

We generalize slightly the notation introduced above. If $H$ is a $g$-invariant subgroup of a group $G$, where $g \in G$ or $g \in \operatorname{Aut} G$, then let

$$
E_{H, n}(g)=\langle[h, \underbrace{g, g, \ldots, g}_{n}] \mid h \in H\rangle \text {. }
$$

Thus, $E_{n}(g)=E_{G, n}(g)$ when $g \in G$ and it is clear from the context which group $G$ is involved. It is clear that $C_{G}(g)$ normalizes $E_{G, n}(g)$.

We are now going to prove Theorem 1.1. The proof reduces to the following proposition.

Proposition 2.2. Let $\alpha$ be an automorphism of a finite soluble group $G$ such that $G=$ $[G, \alpha]$. Let $n$ be a positive integer. Then $E_{G, n}(\alpha)=G$.

Proof. Let $E=E_{G, n}(\alpha)$ for brevity. Let $G$ be a counterexample of minimal order, and let $M$ be a minimal $\alpha$-invariant normal subgroup of $G$. Since the image of $E$ is obviously equal to the similar subgroup constructed for $G / M$, we have $G=M E$ and $M \not E$. Then $M \cap E=1$ by minimality of $M$ because $M$ is abelian and $G=M E$.

Suppose that $C_{M}(\alpha) \neq 1$. Since $C_{M}(\alpha)$ normalizes $E$, we have $\left[C_{M}(\alpha), E\right] \leqslant M \cap E=1$. Then $C_{M}(\alpha)$ is central in $G$ since $M$ is abelian and $G=E M$. By minimality, $C_{M}(\alpha)=M$ and $G=M \times E$. This contradicts the hypothesis $G=[G, \alpha]$.

Thus, $C_{M}(\alpha)=1$. Then the mapping $m \rightarrow[m, \alpha]$ of $M$ into $M$ is injective, and therefore surjective, since $M$ is finite. Hence every element of $M$ has the form $[m, \alpha]$, and therefore also the form $[m, \alpha, \alpha, \ldots, \alpha]$ with $\alpha$ repeated $n$ times. Then $M \leqslant E$, which contradicts the equation $E \cap M=1$. 
Proof of Theorem 1.1. Recall that $g$ is an element of a finite soluble group $G$, and the Fitting height of $E_{n}(g)$ is equal to $k$; we need to show that $g$ belongs to $F_{k+1}(G)$. Consider the subgroups

$$
[\ldots[[G, g], g], \ldots, g]
$$

formed by taking successive commutator subgroups; in particular, these subgroups are subnormal in $G$. Let $H$ be the smallest of the subgroups $[\ldots[[G, g], g], \ldots, g]$. Note that if $H=1$, then $g$ is a left-Engel element and therefore $g \in F(G)$ and there is nothing to prove. In any case, $H=[H, g]$.

Let $N=\left\langle H^{G}\right\rangle$ be the normal closure of $H$. By construction, the image of $g$ in $G / N$ is a left-Engel element and therefore belongs to the Fitting subgroup of $G / N$. If we prove that the Fitting height of $N$ is at most $k$, then $N$, as a normal subgroup, is contained in $F_{k}(G)$ and then $g \in F_{k+1}(G)$, as required. Since $H$ is subnormal in $G$, the Fitting height of its normal closure $N$ is the same as that of $H$ by Lemma 2.1(b). Thus, it suffices to show that the Fitting height of $H$ is at most $k$.

Consider the automorphism $\alpha$ induced on $H$ by $g$ acting by conjugation. The subgroup $E_{H, n}(\alpha)$ is clearly contained in $E_{n}(g)$ and therefore has Fitting height at most $k$. Since $[H, \alpha]=H$, by Proposition 2.2 we obtain $H=E_{H, n}(\alpha)$; hence the result.

\section{DiRECT PRODUCtS OF NONABELIAN FINITE SIMPLE GROUPS}

We begin with elementary consequences of the Schreier conjecture, which may well be known.

Lemma 3.1. If a finite group $G$ has a normal series all of whose factors are nonabelian simple groups, then $G$ is a direct product of nonabelian simple groups.

Proof. Let

$$
1=G_{0} \triangleleft G_{1} \triangleleft G_{2} \triangleleft \cdots \triangleleft G_{j-1} \triangleleft G_{j}=G
$$

be a normal series all of whose factors $G_{i} / G_{i-1}$ are nonabelian simple groups. We use induction on the length of the series $j$, the basis of which is obvious. For $j>1$, the quotient $G / G_{1}$ is a direct product of nonabelian simple groups by the induction hypothesis. The quotient $G / C_{G}\left(G_{1}\right)$ embeds in the automorphism group of $G_{1}$. Since the group of outer automorphisms of $G_{1}$ is soluble by the Schreier conjecture and $G$ has no soluble homomorphic images by the Jordan-Hölder theorem, we must have $G=G_{1} C_{G}\left(G_{1}\right)=$ $G_{1} \times C_{G}\left(G_{1}\right)$, since $G_{1} \cap C_{G}\left(G_{1}\right)=1$. The result follows.

We shall use without special references the well-known fact that in any direct product $S_{1} \times \cdots \times S_{r}$ of nonabelian finite simple groups the only normal subgroups are products $S_{i_{1}} \times \cdots \times S_{i_{l}}$ of some of the factors.

Recall that a subgroup $H$ of a direct product $G_{1} \times \cdots \times G_{r}$ is called a subdirect product of the groups $G_{1}, \ldots, G_{r}$ if the natural projection of $H$ onto every $G_{i}$ is equal to $G_{i}$.

Lemma 3.2. Let $G=S_{1} \times \cdots \times S_{r}$ be a direct product of isomorphic nonabelian finite simple groups. Let $H \leqslant G$ be a subdirect product of the same groups. Then $H=H_{1} \times$ $\cdots \times H_{u}$, where every $H_{i}$ is isomorphic to $S_{1}$.

Proof. Let $T_{i}$ denote the kernel of the $i$ th projection of $H$. Then intersections of some of the $T_{i}$ form a series as in Lemma 3.1.

For the rest of the section we shall work under the following setting. 
Hypothesis 3.3. Let $S=S_{1} \times \cdots \times S_{r}$ be a direct product of $r$ isomorphic finite nonabelian simple groups and let $\varphi$ be the natural automorphism of $S$ of order $r$ that regularly permutes the $S_{i}$.

Note that $S$ has no proper normal $\varphi$-invariant subgroups. In particular, $[S, \varphi]=S$.

Let $D=C_{S}(\varphi)$ be the diagonal. More generally, a subgroup of $S$ that is the diagonal (with respect to $\varphi$ ) in the product of some of the $S_{i}$ (not necessarily of all) is called a $d$-subgroup. More precisely, a $d$-subgroup has the form

$$
\left\{\left(\ldots, a_{i_{1}}, \ldots, a_{i_{2}}, \ldots, a_{i_{l}}, \ldots\right) \mid a_{i_{s}}=a_{i_{1}}^{\varphi^{i_{s}-i_{1}}}\right\},
$$

where dots denote segments of 1 s (possibly empty). In cases where we want to indicate the set of indices $I=\left\{i_{1}, \ldots, i_{l}\right\}$ of nontrivial coordinates in a $d$-subgroup, we call it a $d(I)$-subgroup.

Lemma 3.4. Under Hypothesis 3.3, suppose that $K$ is the $d(I)$-subgroup for some $I$. Then $C_{S}(K)$ is the product of $S_{i}$ with $i \notin I$ and $N_{S}(K)=K \times C_{S}(K)$.

Proof. This follows from the fact that $Z\left(S_{i}\right)=1$. The part about $C_{S}(K)$ is clear. If an element $b$ of $S$ has different coordinates $b_{u} \neq b_{v}$ for $u, v \in I$, then there is $x \in K$ with nontrivial coordinates $a$ such that $a^{b_{u}} \neq a^{b_{v}}$, so that $b \notin N_{S}(K)$.

Lemma 3.5. Under Hypothesis 3.3, suppose that $K$ is a $d$-subgroup and $x \in S$ is such that $K^{x}$ is also a d-subgroup. Then actually $x \in N_{S}(K)$.

Proof. This follows from the fact that conjugation by $x \in S$ does not change the set of indices of nontrivial coordinates.

Lemma 3.6. Under Hypothesis 3.3, let a subgroup $H$ of $S$ be isomorphic to $S_{1}$ and suppose that $H$ is normalized by the diagonal $D$. Then $H$ is a d-subgroup.

Proof. It is sufficient to show that if $H$ has nontrivial projections on some two subgroups $S_{i}$ and $S_{j}$, then the projection $P$ of $H$ onto $S_{i} \times S_{j}$ is equal to the $d(\{i, j\})$-subgroup $K$. If this is not the case, then $P \cap K=1$, since $P$ is normalized by $K$ by hypothesis and $K \cong P \cong S_{1}$. Then $P K=S_{i} \times S_{j}$ and therefore $P$ is normal in $S_{i} \times S_{j}$. Being a proper subgroup, it must be one of the factors, which contradicts the choice of $S_{i}, S_{j}$

Lemma 3.7. Assume Hypothesis 3.3 with $r=|\varphi|$ being a prime. If $H$ is a $\varphi$-invariant subgroup of $S$ containing the diagonal $D$, then either $H=D$ or $H=S$.

Proof. Since $H \geqslant D$, by Lemma 3.2 we have $H=H_{1} \times \cdots \times H_{l}$, where every $H_{i}$ is isomorphic to $S_{1}$. Since $D \leqslant H$ normalizes every $H_{i}$, by Lemma $3.6 H_{i}$ is a $d$-subgroup. We assume that $H \neq D$ and prove that $H=S$. Choose $H_{i} \neq D$. We claim that in fact $H_{i}=S_{j}$ for some $i, j$, which will imply that $H=S$ due to the $\varphi$-invariance. Suppose that $H_{i}$ has more than one nontrivial projections onto the $S_{i}$ - but necessarily less than $|\varphi|$, since $H_{i} \neq D$ and $H_{i}$ is a $d$-subgroup. Since $r=|\varphi|$ is a prime, then we can choose a power of $\varphi$ such that $H_{i}^{\varphi^{w}} \neq H_{i}$ and both $H_{i}^{\varphi^{w}}$ and $H_{i}$ have nontrivial projections onto the same $S_{k}$. The former implies that $H_{i}^{\varphi^{w}}=H_{j} \neq H_{i}$, so that $\left[H_{i}, H_{i}^{\varphi^{w}}\right]=1$, while the latter implies that $\left[H_{i}, H_{i}^{\varphi^{w}}\right] \neq 1$, a contradiction.

Lemma 3.8. Assume Hypothesis 3.3 with $r=|\varphi|$ being a prime. Let $n$ be a positive integer and let $F=\left\langle[x, \underbrace{\varphi, \ldots, \varphi}_{n}] \mid x \in S_{i}\right\rangle$. Then $F=S$. 
Proof. First note that $[F, \varphi] \neq 1$. Indeed, choose some element $x \in S_{1}$ of prime order $q \neq r$. Since $Q=\left\langle x^{\langle\varphi\rangle}\right\rangle$ is an abelian $\varphi$-invariant $q$-group, we have

$$
1 \neq[Q, \varphi]=[[Q, \varphi], \varphi]=\{[x, \underbrace{\varphi, \varphi, \ldots, \varphi}_{k}] \mid k \geqslant n+1\} .
$$

In particular, $1 \neq[x, \underbrace{\varphi, \varphi, \ldots, \varphi}_{n+1}] \in[F, \varphi]$. It follows from the definition that $F$ is a $\varphi$ invariant subgroup, which is also normalized by $D$. Then $F D$ is a $\varphi$-invariant subgroup properly containing $D$ and therefore $F D=S$ by Lemma 3.7. As a result, $F$ is normal in $S$. Being nontrivial and $\varphi$-invariant, it must coincide with $S$.

Lemma 3.9. Assume Hypothesis 3.3. Let $n$ be a positive integer and let $E=E_{S, n}(\varphi)=$ $\langle[x, \underbrace{\varphi, \ldots, \varphi}_{n}] \mid x \in S\rangle$. Then $E=S$.

Proof. Let $\varphi^{k}$ be of prime order. Then $G=\left\langle[g, \underbrace{\varphi^{k}, \ldots, \varphi^{k}}_{n}] \mid g \in S_{i}, i=1, \ldots, r\right\rangle$ by Lemma 3.8 applied to each orbit of $\varphi^{k}$. It remains to observe that $[g, \underbrace{\varphi^{k}, \ldots, \varphi^{k}}_{n}] \in E$ for $g \in S_{i}$. Indeed, $A=\left\langle g^{\langle\varphi\rangle}\right\rangle$ is an abelian $\varphi$-invariant group, in which repeated application of the formulae $\left[b, \varphi^{i+1}\right]=\left[b, \varphi^{i}\right][b, \varphi]$ and $\left[b c, \varphi^{i}\right]=\left[b, \varphi^{i}\right]\left[c, \varphi^{i}\right]$ for $b, c \in A$ expresses the commutator $[g, \underbrace{\varphi^{k}, \ldots, \varphi^{k}}_{n}]$ in terms of commutators $[x, \underbrace{\varphi, \ldots, \varphi}_{n}]$ for $x \in A$.

\section{ORBits IN SOME TRANSITIVE PERMUtATional ACTIONS}

We shall need several lemmas on transitive permutational actions of certain finite groups $G$ concerning the existence of exact (regular) orbits of an element $g \in G$. Rather than speaking about orbits, we prefer to state these lemmas in terms of intersections of $\langle g\rangle$ with a conjugate of the stabilizer of a point $H \leqslant G$. The following lemma is quite elementary.

Lemma 4.1. Let $g$ be an element, and $H$ a subgroup of a group $G$. Suppose that $g$ is contained in a subgroup $G_{0} \leqslant G$ and $\langle g\rangle$ has trivial intersection with $\left(H \cap G_{0}\right)^{x}$ for some $x \in G_{0}$. Then $H^{x} \cap\langle g\rangle=1$.

Proof. Let $H_{0}=H \cap G_{0}$. Then

$$
H^{x} \cap\langle g\rangle=H^{x} \cap G_{0} \cap\langle g\rangle=\left(H \cap G_{0}\right)^{x} \cap\langle g\rangle=H_{0}^{x} \cap\langle g\rangle=1 .
$$

Lemma 4.2. Let $S=S_{1} \times \cdots \times S_{l}$ be a direct product of finitely many nonabelian finite simple groups. Let $\varphi$ be an automorphism of $S$ such that every $\varphi$-orbit of the permutational action on $\left\{S_{1}, \ldots, S_{l}\right\}$ has $|\varphi|$ elements. Let $H$ be a subgroup of $S\langle\varphi\rangle$ such that $S \mathbb{*} H$. Then there is $x \in S$ such that $H^{x} \cap\langle\varphi\rangle=1$.

Proof. We proceed by induction on $|S\langle\varphi\rangle|$. As a basis of induction we can consider the case where $|\varphi|$ is a prime, where the result is obvious. Indeed, if $\varphi \in \bigcap_{x \in S} H^{x}$, then, since $\bigcap_{x \in S} H^{x}$ is $S$-invariant,

$$
S=[S, \varphi] \leqslant\left[S, \bigcap_{x \in S} H^{x}\right] \leqslant H
$$

a contradiction with hypothesis. Next, if the order of $\varphi$ is divisible by $p^{2}$ for some prime $p$, then we can work with $\varphi^{p}$, which also satisfies the condition that all its orbits are of length $\left|\varphi^{p}\right|$. By induction applied to $S\left\langle\varphi^{p}\right\rangle$ and $H_{0}=H \cap S\left\langle\varphi^{p}\right\rangle$ we obtain $x \in S$ such that $H_{0}^{x} \cap\left\langle\varphi^{p}\right\rangle=1$, whence $H^{x} \cap\left\langle\varphi^{p}\right\rangle=1$ by Lemma 4.1 and then $H^{x} \cap\langle\varphi\rangle=1$. So 
we can assume that the order of $\varphi$ is square-free and is divisible by at least two primes. Let $p$ be a prime divisor of $|g|$. Let $\beta=\varphi^{p}$ and let $\alpha$ be an element of order $p$ in $\langle\varphi\rangle$. By induction there exists $x \in S$ such that $(H \cap S\langle\beta\rangle)^{x} \cap\langle\beta\rangle=1$, whence $H^{x} \cap\langle\beta\rangle=1$. If $\alpha \notin H^{x}$, then the proof is complete. Therefore we can assume that $\alpha \in H^{x}$. Replacing $H$ by $H^{x}$ we now assume that $H \cap\langle\beta\rangle=1$ and $\alpha \in H$.

We can choose a $\varphi$-orbit such that the product $T$ of the simple factors in this orbit satisfies $T \nless H$. If $T \neq S$, then by induction there is $x \in T$ such that $(H \cap T\langle\varphi\rangle)^{x} \cap\langle\varphi\rangle=1$; then $H^{x} \cap\langle\varphi\rangle=1$ by Lemma 4.1. Thus, we can assume that $S$ is a product over one $\varphi$-orbit. Let $C=C_{S}(\beta)$. Since $H^{x} \cap\langle\beta\rangle=1$ for all $x \in C$, we can assume that $\alpha^{x} \in H$ for all $x \in C$. Since $\alpha^{x}=\alpha[\alpha, x]$ and $\alpha \in H$, we deduce that $[C, \alpha] \leqslant H$. Since $C$ is the direct product of $d$-subgroups corresponding to $\beta$-orbits, and these $d$-subgroups are regularly permuted by $\alpha$, it follows that $C=[C, \alpha]$. In particular, the diagonal $D$ of $S$ is contained in $H$ and so $H \cap S$ is a subdirect product of the $S_{i}$. Hence $H \cap S$ is a direct product of subgroups $H_{i}$ isomorphic to $S_{1}$ by Lemma 3.2.

If $S H \neq S\langle\varphi\rangle$, then $S H=S\left\langle\varphi^{t}\right\rangle$ for some $t>1$. In this case we can apply the induction hypothesis to $S\left\langle\varphi^{t}\right\rangle$, find $x \in S$ such that $H^{x} \cap\left\langle\varphi^{t}\right\rangle=1$, and then also $H^{x} \cap\langle\varphi\rangle=H^{x} \cap S\left\langle\varphi^{t}\right\rangle \cap\langle\varphi\rangle=H^{x} \cap\left\langle\varphi^{t}\right\rangle=1$, since $S \cap\langle\varphi\rangle=1$. Thus, we assume that $S H=S\langle\varphi\rangle$. Then $s \varphi=h$ for some $s \in S$ and $h \in H$. Note that $h$ induces the same permutation on $\left\{S_{1}, \ldots, S_{l}\right\}$ as $\varphi$.

Since $D \leqslant H$ normalizes every $H_{i}$, by Lemma 3.6 we obtain that $H_{i}$ is a $d$-subgroup. We claim that in fact $H_{i}=S_{j}$ for some $j$. Indeed, choose an element $a_{1} \in S_{1}$ such that $a_{1} \neq a_{1}^{-1}$. By assumption, $H^{a_{1}^{-1}} \cap\langle\varphi\rangle \neq 1$, so that there is a nontrivial element $\psi \in\langle\varphi\rangle$ such that $\psi^{a_{1}} \in H$. Then for every $i$ we have $H_{i}^{\psi^{a_{1}}}=H_{j}$ for some $j=j(i)$. Since $H_{i}^{\psi}$ is also a $d$-subgroup and $\left(H_{i}^{\psi}\right)^{\left[\psi, a_{1}\right]}=H_{i}^{\psi^{a_{1}}}=H_{j}$, by Lemma 3.5 we obtain that $\left[\psi, a_{1}\right]$ normalizes $H_{i}^{\psi}=H_{j}$. Thus, $\psi$ permutes the subgroups $H_{u}$. It follows that $H_{j}^{\left[\psi, a_{1}\right]}=H_{j}$ for all $j$. Since $D \leqslant H$, there is a $d$-subgroup $H_{j}$ with nontrivial projections onto at least one of the factors $S_{1}$ or $S_{k}=S_{1}^{\psi} \neq S_{1}$, which contain the nontrivial projections $a_{1}$ and $\left(a_{1}^{-1}\right)^{\psi}$ of $\left[\psi, a_{1}\right]$. Since $a_{1} \neq a_{1}^{-1}$, it follows from Lemma 3.4 that $H_{j}=S_{1}$ or $H_{j}=S_{k}$.

The latter implies that $S \leqslant H$ due to the invariance under $h \in H$, which induces the same permutation on the $S_{u}$ as $\varphi$. This contradicts the hypothesis.

Lemma 4.3. Let $S=S_{1} \times \cdots \times S_{r}$ be a direct product of $r$ isomorphic nonabelian finite simple groups, and let $\varphi$ be an automorphism of $S$ that transitively permutes the $S_{i}$. Suppose that for some prime $p$ the stabilizer of $S_{1}$ in $\langle\varphi\rangle$ is a p-subgroup, possibly trivial. Let $H$ be a subgroup of $S\langle\varphi\rangle$ such that $S \notin H$. Then there is $x \in S$ such that $H^{x} \cap\langle\varphi\rangle=1$.

Note that we do not exclude the case of $r=1$, when of course $\varphi$ is an automorphism of $S=S_{1}$ of prime-power order.

Remark 4.4. The condition in Lemma 4.3 that the stabilizer of $S_{1}$ in $\langle\varphi\rangle$ is a $p$-subgroup is essential. The smallest example is given by the alternating group $S_{1}=A l t_{5}$, its automorphism $\varphi$ induced by conjugation by an element of order 6 in the ambient symmetric group $S_{y m_{5}}$, and $H$ being a stabilizer of a point in the natural representation of $S_{y} m_{5}$ on 5 points.

Proof of Lemma 4.3. We proceed by induction on $|S\langle\varphi\rangle|$. As a basis of induction we can consider the case where $|\varphi|$ is a prime, where the result is obvious. Indeed, if $\varphi \in \bigcap_{x \in S} H^{x}$, then, since $\bigcap_{x \in S} H^{x}$ is $S$-invariant, $S=[S, \varphi] \leqslant\left[S, \bigcap_{x \in S} H^{x}\right] \leqslant H$, a contradiction with hypothesis. 
Next, if the order of $\varphi$ is divisible by $q^{2}$ for some prime $q$, then we can work with $\varphi^{q}$ in place of $\varphi$. There is an orbit (possibly one-element) of the permutation induced by $\varphi^{q}$ on the set $\left\{S_{1}, \ldots, S_{r}\right\}$ such that the product $T$ over this orbit satisfies $T \& H$. Then the group $T\left\langle\varphi^{q}\right\rangle$ satisfies the hypotheses of the lemma with $H_{0}=H \cap T\left\langle\varphi^{q}\right\rangle$ in place of $H$. By induction we find $x \in T$ such that $H_{0}^{x} \cap\left\langle\varphi^{q}\right\rangle=1$, whence $H_{0}^{x} \cap\langle\varphi\rangle=1$, and then $H^{x} \cap\langle\varphi\rangle=1$ by Lemma 4.1. So we can assume that the order of $\varphi$ is square-free and is divisible by at least two primes.

If the Sylow $p$-subgroup of $\langle\varphi\rangle$ is trivial, then we get the result by Lemma 4.2. So let $\alpha$ be an element of order $p$ which generates the stabilizer of $S_{1}$ in $\langle\varphi\rangle$, and let $\beta=\varphi^{p}$. By induction there exists $x \in S$ such that $(H \cap S\langle\beta\rangle)^{x} \cap\langle\beta\rangle=1$, whence $H^{x} \cap\langle\beta\rangle=1$ by Lemma 4.1. If $\alpha \notin H^{x}$, then the proof is complete; so we can assume that $\alpha \in H^{x}$. Replacing $H$ by $H^{x}$ we now assume that $H \cap\langle\beta\rangle=1$ and $\alpha \in H$.

Let $D=C_{S}(\beta)$. Since $H^{x} \cap\langle\beta\rangle=1$ for all $x \in D$, we can assume that $\alpha^{x} \in H$ for all $x \in D$. Since $\alpha^{x}=\alpha[\alpha, x]$ and $\alpha \in H$, we deduce that $D=[D, \alpha] \leqslant H$. Hence $H \cap S$ is a subdirect product of the $S_{i}$. By Lemma 3.2 we obtain that $H \cap S$ is a direct product of subgroups $H_{i}$ isomorphic to $S_{1}$.

If $S H \neq S\langle\varphi\rangle$, then $S H=S\left\langle\varphi^{t}\right\rangle$ for some $t>1$. We can choose an orbit of the permutation induced by $\varphi^{t}$ on the set $\left\{S_{1}, \ldots, S_{r}\right\}$ such that the product $T$ of the simple factors in this orbit satisfies $T \nless H$. The group $T\left\langle\varphi^{t}\right\rangle$ satisfies the hypotheses of the lemma with $H_{0}=H \cap T\left\langle\varphi^{t}\right\rangle$ in place of $H$. By induction we find $x \in T$ such that $H_{0}^{x} \cap\left\langle\varphi^{t}\right\rangle=1$, whence $H^{x} \cap\left\langle\varphi^{t}\right\rangle=1$ by Lemma 4.1, and then $H^{x} \cap\langle\varphi\rangle=H^{x} \cap S\left\langle\varphi^{t}\right\rangle \cap\langle\varphi\rangle=H^{x} \cap\left\langle\varphi^{t}\right\rangle=1$, since $S \cap\langle\varphi\rangle=1$. Thus, we assume that $S H=S\langle\varphi\rangle$. Then $s \varphi=h$ for some $s \in S$ and $h \in H$. Note that $h$ induces the same permutation on $\left\{S_{1}, \ldots, S_{r}\right\}$ as $\varphi$.

Since $D \leqslant H$, it follows that $D$ normalizes every $H_{i}$, and by Lemma 3.6 we obtain that every $H_{i}$ is a $d$-subgroup. If $D=H \cap S$, then $D$ is normalized by $s \varphi=h \in H$. Since $D$ is also normalized by $\varphi$, we obtain that $D$ is normalized by $s$, whence $s \in D$ by Lemma 3.4. Since $D \leqslant H$, we obtain that $\varphi \in H$, a contradiction with our assumption. Therefore we can assume that there is $H_{i} \neq D$.

We claim that in fact $H_{i}=S_{j}$ for some $j$. Indeed, choose an element $a_{1} \in S_{1}$ such that $a_{1} \neq a_{1}^{-1}$. By assumption, $H^{a_{1}^{-1}} \cap\langle\varphi\rangle \neq 1$, so that there is a nontrivial element $\psi \in\langle\varphi\rangle$ such that $\psi^{a_{1}} \in H$. If $\psi \notin\langle\alpha\rangle$, then we argue in the same fashion as in the proof of Lemma 4.2. Namely, $H_{i}^{\psi}$ is a $d$-subgroup for every $i$ and $\left(H_{i}^{\psi}\right)^{\left[\psi, a_{1}\right]}=H_{i}^{\psi^{a_{1}}}=H_{j}$; hence $\left(H_{i}^{\psi}\right)^{\left[\psi, a_{1}\right]}=H_{i}^{\psi}$ by Lemma 3.5; therefore $H_{j}^{\left[\psi, a_{1}\right]}=H_{j}$ for all $j$. Since $D \leqslant H$, there is a $d$-subgroup $H_{j}$ with nontrivial projections onto at least one of the factors $S_{1}$ or $S_{k}=S_{1}^{\psi} \neq S_{1}$, which contain $a_{1}$ and $\left(a_{1}^{-1}\right)^{\psi}$. Since $a_{1} \neq a_{1}^{-1}$, it follows from Lemma 3.4 that $H_{j}=S_{1}$ or $H_{j}=S_{k}$, which leads to $S \leqslant H$, a contradiction.

It remains to consider the case where $1 \neq H^{a_{1}^{-1}} \cap\langle\varphi\rangle \leqslant\langle\alpha\rangle$ for all $a_{1} \in S_{1}$ such that $\left|a_{1}\right| \neq 2$. Since such elements $a_{1}$ generate $S_{1}$ and $\alpha$ is a nontrivial automorphism of $S_{1}$, there is $a_{1} \in S_{1}$ such that $a_{1} \neq a_{1}^{\alpha}$. Since $\alpha \in H$ and $\alpha^{a_{1}} \in H$ by our assumption, we obtain a nontrivial element $\left[\alpha, a_{1}\right] \in S_{1} \cap H$. Since $H \cap S$ is a direct product of $d$-subgroups $H_{i}$, we must have $S_{1}=H_{j}$ for some $j$.

In any case, we have $H_{i}=S_{j}$ for some $i, j$. This implies that $H=S$ due to the invariance under $h \in H$, which induces the same permutation on the $S_{u}$ as $\varphi$. This contradicts the hypothesis.

Let $N$ be a normal subgroup of a group $G$, and $A$ a subgroup of $G / N$. We shall be saying for brevity that $B$ covers $A$ if $B N / N \geqslant A$.

The following proposition is the main result of this section. 
Proposition 4.5. Let $G\langle g\rangle$ be a finite group with a normal subgroup $G$ and a cyclic subgroup $\langle g\rangle$, and let $R$ be the soluble radical of $G$. Let $\left\langle g_{0}\right\rangle=C_{\langle g\rangle}(G / R)$, so that the image $\bar{g}$ of $g$ in $\langle g\rangle /\left\langle g_{0}\right\rangle$ is the automorphism of $G / R$ induced by conjugation by $g$. Suppose that the following conditions hold:

(1) $G$ is a minimal $g$-invariant subgroup covering $G / R$;

(2) $G \cap\langle g\rangle \leqslant C_{\langle g\rangle}(G / R)$;

(3) $G / R=S_{1} \times \cdots \times S_{r}$ is a direct product of isomorphic nonabelian finite simple groups, which are transitively permuted by $\bar{g}$;

(4) for some prime $p$ the stabilizer of $S_{1}$ in $\langle\bar{g}\rangle$ is a p-subgroup, possibly trivial.

Then for any subgroup $H \leqslant G\langle g\rangle$ such that $G \notin H$ there is $z \in G$ such that $H^{z} \cap\langle g\rangle \leqslant$ $\left\langle g_{0}\right\rangle$.

For brevity we shall refer to orbits of elements of $G\langle g\rangle$ on $\left\{S_{i}\right\}$ meaning orbits in the set $\left\{S_{1}, \ldots, S_{r}\right\}$ of the corresponding induced permutations.

Proof. Induction on $|G\langle g\rangle|$. As a basis of induction we can take the case $R=1$. In this case, $\left\langle g_{0}\right\rangle$ is a central subgroup, and in the quotient $G\langle g\rangle /\left\langle g_{0}\right\rangle$ the image of $H$ does not contain the image of $G$. Indeed, otherwise $G \leqslant H\left\langle g_{0}\right\rangle$ and then $G=[G, G] \leqslant$ $\left[H\left\langle g_{0}\right\rangle, H\left\langle g_{0}\right\rangle\right]=[H, H]$, contrary to the assumption $G \not H$. Thus, due to conditions (3), (4), we can apply Lemma 4.3 to the group $G\langle g\rangle /\left\langle g_{0}\right\rangle$ and find $z \in G$ such that $H^{z} \cap\langle g\rangle \leqslant\left\langle g_{0}\right\rangle$, as required.

Now let $R \neq 1$ and let $M$ be a minimal $g$-invariant abelian normal subgroup of $G$. If $G \nless M H$, then we can apply induction to $G\langle g\rangle / M$. Indeed, since $M \leqslant R$, conditions (1), (3), (4) obviously hold for the images in $G\langle g\rangle / M$, and the image of $\left\langle g_{0}\right\rangle$ is the centralizer of $G / R$ in the image of $\langle g\rangle$. We check condition (2): if $a m=g^{i}$ for $a \in G, m \in M$, then $g^{i} \in G$ and therefore $g^{i} \in C_{\langle g\rangle}(G / R)$ by condition (2) for $G\langle g\rangle$. By induction we find $z \in G$ such that $H^{z} \cap\langle g\rangle \leqslant\left\langle g_{0}\right\rangle M$; then $H^{z} \cap\langle g\rangle \leqslant\left\langle g_{0}\right\rangle M \cap\langle g\rangle \leqslant C_{\langle g\rangle}(G / R)=\left\langle g_{0}\right\rangle$, as required. Thus, we can assume that $G \leqslant M H$.

Suppose that $\left\langle g_{0}\right\rangle=C_{\langle g\rangle}(G / R)$ contains some nontrivial Sylow $q$-subgroup $\left\langle g_{q}\right\rangle$ of $\langle g\rangle$. Then $\langle g\rangle=\left\langle g_{1}\right\rangle \times\left\langle g_{q}\right\rangle$, where $\left\langle g_{1}\right\rangle$ is (necessarily nontrivial) Hall $q^{\prime}$-subgroup. We choose a minimal $g_{1}$-invariant subgroup $G_{1}$ of $G$ covering $G / R$. Then the subgroups $G_{1}^{g_{q}^{j}}$ are of similar nature and together generate $G$ by minimality of $G$. Since $G \nless H$, we can assume without loss of generality that $G_{1} \nless H$. The hypotheses of the proposition are satisfied for $G_{1}\left\langle g_{1}\right\rangle$ and $H \cap G_{1}\left\langle g_{1}\right\rangle$. Indeed, (1) is true by construction, (2) is inherited from the same condition for $g$, while (3) and (4) hold because the action of $g_{1}$ on $G_{1} / R_{1}$ is similar to the action of $g$ on $G / R$. Since $G_{1} \nless H \cap G_{1}\left\langle g_{1}\right\rangle$, by induction we find $z \in G_{1}$ such that $\left(H \cap G_{1}\left\langle g_{1}\right\rangle\right)^{z} \cap\left\langle g_{1}\right\rangle \leqslant\left\langle g_{0}\right\rangle$, whence $H^{z} \cap\left\langle g_{1}\right\rangle \leqslant\left\langle g_{0}\right\rangle$. Then, since $\left\langle g_{q}\right\rangle \leqslant\left\langle g_{0}\right\rangle$ by assumption,

$$
H^{z} \cap\langle g\rangle=\left(H^{z} \cap\left\langle g_{1}\right\rangle\right) \times\left(H^{z} \cap\left\langle g_{q}\right\rangle\right) \leqslant\left\langle g_{0}\right\rangle .
$$

Thus, we can assume that every Sylow $q$-subgroup of $\langle g\rangle$ acts nontrivially on $G / R$.

Now suppose that $G H \neq G\langle g\rangle$; then $G H=G\left\langle g^{s}\right\rangle$ for some prime $s$. Note that $\left\langle g^{s}\right\rangle \geqslant\left\langle g_{0}\right\rangle$ by the assumption at the end of the last paragraph. For a $g^{s}$-orbit on $\left\{S_{i}\right\}$, we choose a minimal $g^{s}$-invariant subgroup $G_{1}$ of $G$ covering the product of the $S_{i}$ in this $g^{s}$-orbit. Then the subgroups $G_{1}^{g^{j}}$ are of similar nature and $G=\left\langle G_{1}, G_{1}^{g}, \ldots, G_{1}^{g^{s-1}}\right\rangle$ by minimality of $G$. Since $G \nless H$, we can assume without loss of generality that $G_{1} \nless H$. Note that the centralizer of $G_{1} R / R$ in $\left\langle g^{s}\right\rangle$ is equal to $\left\langle g_{0}\right\rangle$ because the products of simple factors over $g^{s}$-orbits are permuted by $g$. The group $G_{1}\left\langle g^{s}\right\rangle$ satisfies the hypotheses of the proposition with $H_{1}=H \cap G_{1}\left\langle g^{s}\right\rangle$ in place of $H$. Indeed, conditions (1), (3) are 
satisfied by construction of $G_{1}$, and conditions (2), (4) are obviously inherited from the same conditions for $\langle g\rangle$.

By induction we find $z \in G_{1}$ such that $H_{1}^{z} \cap\left\langle g^{s}\right\rangle \leqslant\left\langle g_{0}\right\rangle$. Note that $G\left\langle g^{s}\right\rangle \cap\langle g\rangle=\left\langle g^{s}\right\rangle$, since $\left\langle g^{s}\right\rangle \geqslant\left\langle g_{0}\right\rangle$ and $G \cap\langle g\rangle \leqslant\left\langle g_{0}\right\rangle$ by condition (2). Then

$$
\begin{aligned}
H^{z} \cap\langle g\rangle & =H^{z} \cap G\left\langle g^{s}\right\rangle \cap\langle g\rangle=H^{z} \cap\left\langle g^{s}\right\rangle \\
& =H^{z} \cap G_{1}\left\langle g^{s}\right\rangle \cap\left\langle g^{s}\right\rangle=\left(H \cap G_{1}\left\langle g^{s}\right\rangle\right)^{z} \cap\left\langle g^{s}\right\rangle \\
& =H_{1}^{z} \cap\left\langle g^{s}\right\rangle \leqslant\left\langle g_{0}\right\rangle
\end{aligned}
$$

Thus, we can assume that $G H=G\langle g\rangle$.

It follows that $M \cap H=1$ by minimality of $M$. Indeed, this intersection is normal in $M H$ because $M$ is abelian, and $M H=G\langle g\rangle$ because $M H \geqslant G H=G\langle g\rangle$. We also have $M \not H$, since $G \not H$.

Let $a_{1}, \ldots, a_{s}$ be elements of prime orders in $\langle g\rangle$, one for each prime divisor of $|g|$. Suppose that for some $i_{0}$ we have $a_{i_{0}}^{x} \notin H$ for every $x \in G$, and let $\left|a_{i_{0}}\right|=q$. Let $\left\langle g_{1}\right\rangle$ be the Hall $q^{\prime}$-subgroup of $\langle g\rangle$. We can assume that $g_{1}$ acts nontrivially on $G / R$, for otherwise $\left\langle g_{1}\right\rangle \leqslant\left\langle g_{0}\right\rangle$, and then $H \cap\langle g\rangle \leqslant\left\langle g_{0}\right\rangle$ and we are done. The products of simple factors of $G / R$ over $g_{1}$-orbits are transitively permuted by $g$. Hence $g_{1}$ acts nontrivially on each such product, and the centralizer of such a product in $\left\langle g_{1}\right\rangle$ is equal to $\left\langle g_{1}\right\rangle \cap\left\langle g_{0}\right\rangle$. As above, since $G \nless H$, we can find a $g_{1}$-orbit on $\left\{S_{i}\right\}$ such that $H$ does not contain a minimal $g_{1}$-invariant subgroup $G_{1}$ of $G$ covering the product of the $S_{i}$ in this $g_{1}$-orbit. Then the hypotheses of the proposition hold for $G_{1}, g_{1}$, and $H_{1}=H \cap G_{1}\left\langle g_{1}\right\rangle$ in place of $G, g$, and $H$. Indeed, conditions (1), (3) hold by construction, condition (4) is inherited from the same condition for $g$, and condition (2) follows from the same condition for $g$ because an element in $\left\langle g_{1}\right\rangle$ centralizing $G_{1} R / R$ also centralizes $G / R$. By induction we find $z \in G_{1}$ such that $H_{1}^{z} \cap\left\langle g_{1}\right\rangle \leqslant\left\langle g_{0}\right\rangle$, and therefore also

$$
H^{z} \cap\left\langle g_{1}\right\rangle=H^{z} \cap G_{1}\left\langle g_{1}\right\rangle \cap\left\langle g_{1}\right\rangle=\left(H \cap G_{1}\left\langle g_{1}\right\rangle\right)^{z} \cap\left\langle g_{1}\right\rangle=H_{1}^{z} \cap\left\langle g_{1}\right\rangle \leqslant\left\langle g_{0}\right\rangle .
$$

Since $a_{i_{0}}^{z^{-1}} \notin H$ by our assumption, we obtain $H^{z} \cap\langle g\rangle \leqslant\left\langle g_{0}\right\rangle$. Thus, we can assume that for every $a_{i}$ there is $x \in G$ such that $a_{i}^{x} \in H$. Since $G \leqslant M H$, for every $i$ we can choose $m_{i} \in M$ such that $a_{i}^{m_{i}} \in H$.

We set

$$
M_{i}=\left\{m \in M \mid a_{i}^{m} \in H\right\} .
$$

Since $M$ is abelian, a direct calculation shows that $M_{i}=m_{i} C_{M}\left(a_{i}\right)$. Indeed, if $a_{i}^{m} \in H$, then $a_{i}^{m}\left(a_{i}^{-1}\right)^{m_{i}} \in M \cap H=1$, whence $a_{i}^{m m_{i}^{-1}} a_{i}^{-1}=1$, which means that $m \in m_{i} C_{M}\left(a_{i}\right)$. Thus, $M_{i} \subseteq m_{i} C_{M}\left(a_{i}\right)$. The reverse inclusion is obvious: $a_{i}^{m m_{i}}=a_{i}^{m_{i}} \in H$ for any $m \in C_{M}\left(a_{i}\right)$.

If there is $z \in M \backslash \bigcup M_{i}$, then $a_{i}^{z} \notin H$ for all $i$, so that $H^{z^{-1}} \cap\langle g\rangle=1$ and the proof is complete. Therefore we can assume that $M=\bigcup M_{i}$.

Suppose that $M=M_{i_{0}}$ for some $i_{0}$. Then $M=C_{M}\left(a_{i_{0}}\right)$, which implies that $a_{i_{0}} \in H$ and, moreover, $a_{i_{0}}^{x} \in H$ for any $x \in G\langle g\rangle$, since $G \leqslant M H$ and $g$ centralizes $a_{i_{0}}$. In other words,

$$
a_{i_{0}} \in K:=\bigcap_{x \in G\langle g\rangle} H^{x}
$$

where $K$ is a normal subgroup of $G\langle g\rangle$. It now follows that $a_{i_{0}} \in\left\langle g_{0}\right\rangle$ : otherwise, $\left[G / R, a_{i_{0}}\right]=G / R$ and $\left[G, a_{i_{0}}\right] \leqslant[G, K] \leqslant K$, so that $K \cap G$ covers $G / R$ contrary to minimality of $G$, since $G \nless H \geqslant K$.

First suppose that $K_{0}=K \cap R \neq 1$. Then the hypotheses of the proposition hold for the images in $G\langle g\rangle / K_{0}$, which we denote by tilde. Indeed, $\tilde{G} / \tilde{R} \cong G / R$ and $C_{\langle\tilde{g}\rangle}(\tilde{G} / \tilde{R})=\left\langle\tilde{g}_{0}\right\rangle$. 
Conditions (1), (3), (4) obviously hold since $K_{0} \leqslant R$. We check condition (2): if $a k=g^{i}$ for $a \in G, k \in K_{0}$, then $g^{i} \in\left\langle g_{0}\right\rangle$ by condition (2) for $G\langle g\rangle$. Also, $\tilde{G} \& \tilde{H}$ since $K_{0} \leqslant G \cap H$. By induction we find $\tilde{z} \in \tilde{G}$ such that $\tilde{H}^{\tilde{z}} \cap\langle\tilde{g}\rangle \leqslant\left\langle\tilde{g}_{0}\right\rangle$. Then for a pre-image $z \in G$ we have

as required.

$$
H^{z} \cap\langle g\rangle \leqslant\left\langle g_{0}\right\rangle K_{0} \cap\langle g\rangle \leqslant C_{\langle g\rangle}(G / R)=\left\langle g_{0}\right\rangle,
$$

Thus we can assume that $K \cap R=1$. Then $\left[a_{i_{0}}, G\right] \leqslant K \cap R=1$, so $a_{i_{0}}$ is central in $G\langle g\rangle$. We are going to apply induction to $G\langle g\rangle /\left\langle a_{i_{0}}\right\rangle$, where the images are denoted by tilde. Clearly, $\tilde{G} / \tilde{R} \cong G / R$. We claim that $C_{\langle\tilde{g}\rangle}(\tilde{G} / \tilde{R})=\left\langle\tilde{g}_{0}\right\rangle$. Indeed, if $\left[G, g^{i}\right] \leqslant R\left\langle a_{i_{0}}\right\rangle$, then $\left[\left[G, g^{i}\right], g^{i}\right] \leqslant\left[R\left\langle a_{i_{0}}\right\rangle, g^{i}\right] \leqslant R$, whence $\left[G, g^{i}\right] \leqslant R$, since then the image of $g^{i}$ in $G\langle g\rangle / R$ belongs to the Fitting subgroup by Baer's theorem. We now verify the other hypotheses of the proposition. Condition (1): if $\tilde{G}_{1} \leqslant \tilde{G}$ for a $g$-invariant subgroup $\tilde{G}_{1}$ covering $\tilde{G} / \tilde{R}$, then for the full inverse image we have $G_{1} \leqslant G\left\langle a_{i_{0}}\right\rangle$. Then $\left[G_{1}, G_{1}\right] \leqslant\left[G\left\langle a_{i_{0}}\right\rangle, G\left\langle a_{i_{0}}\right\rangle\right] \leqslant G$. Since $\left[G_{1}, G_{1}\right]$ also covers $G / R$, by minimality of $G$ we must have $\left[G_{1}, G_{1}\right]=G$, whence $\tilde{G}_{1}=\tilde{G}$. Condition (2): if $b=g^{i} a_{i_{0}}^{j}$ for $b \in G$, then $g^{i} a_{i_{0}}^{j} \in\left\langle g_{0}\right\rangle$ by condition (2) for $G\langle g\rangle$, whence $g^{i} \in\left\langle g_{0}\right\rangle$, since $a_{i_{0}} \in\left\langle g_{0}\right\rangle$. Conditions (3) and (4) obviously follow from the same conditions for $G\langle g\rangle$. Finally, $\tilde{G} \leftleftarrows \tilde{H}$, since otherwise $G \leqslant H\left\langle a_{i_{0}}\right\rangle=H$, contrary to the hypothesis. By induction we find $z \in G$ such that $H^{z} \cap\langle g\rangle \leqslant\left\langle g_{0}\right\rangle\left\langle a_{i_{0}}\right\rangle=\left\langle g_{0}\right\rangle$.

Thus, we can assume that $M_{i} \neq M$ for every $i$.

Consider the subgroup $A=\left\langle a_{1}^{m_{1}}, \ldots, a_{s}^{m_{s}}\right\rangle \leqslant H$. Since $H \cap M=1$, we have $A \cong$ $A M / M \cong\left\langle a_{1}, \ldots, a_{s}\right\rangle$, which is a cyclic group (a subgroup of $\langle g\rangle$ ). The $\operatorname{subgroup} M$ is an elementary abelian $q$-group for some prime $q$. Let $B$ be a Hall $q^{\prime}$-subgroup of $A$ (possibly, $B=A$ ). In the semidirect product $M A=M\left\langle a_{1}, \ldots, a_{s}\right\rangle$, the Hall $q^{\prime}$-subgroup of $\left\langle a_{1}, \ldots, a_{s}\right\rangle$, which is also a Hall $q^{\prime}$-subgroup of $M A$, is conjugate to $B$ by an element $y \in M$. Since $B \leqslant H$, this means that for every $i$ such that $\left|a_{i}\right| \neq q$ we can replace all those elements $m_{i}$ by this element $y \in m_{i} C_{M}\left(a_{i}\right)$. Without loss of generality, suppose that all the elements $a_{2}, \ldots, a_{s}$ are of order coprime to $q$, while $\left|a_{1}\right|$ may be equal to $q$, or not. If $\left|a_{1}\right| \neq q$, then

$$
M=y C_{M}\left(a_{1}\right) \cup y C_{M}\left(a_{2}\right) \cup \cdots \cup y C_{M}\left(a_{s}\right),
$$

whence, after multiplying by $y^{-1}$,

$$
M=C_{M}\left(a_{1}\right) \cup C_{M}\left(a_{2}\right) \cup \cdots \cup C_{M}\left(a_{s}\right) .
$$

If $\left|a_{1}\right|=q$, then

$$
M=m_{1} C_{M}\left(a_{1}\right) \cup y C_{M}\left(a_{2}\right) \cup \cdots \cup y C_{M}\left(a_{s}\right),
$$

whence, after multiplying by $y^{-1}$,

$$
M=y^{-1} m_{1} C_{M}\left(a_{1}\right) \cup C_{M}\left(a_{2}\right) \cup \cdots \cup C_{M}\left(a_{s}\right) .
$$

Suppose that $y^{-1} m_{1} C_{M}\left(a_{1}\right) \neq C_{M}\left(a_{1}\right)$. In this case, let $b=a_{2} \cdots a_{s}$. If $b$ does not normalize the coset $y^{-1} m_{1} C_{M}\left(a_{1}\right)$, then (4.2) implies

$$
\left(y^{-1} m_{1} C_{M}\left(a_{1}\right)\right)^{b} \subseteq C_{M}\left(a_{2}\right) \cup \cdots \cup C_{M}\left(a_{s}\right)
$$

and then also

$$
y^{-1} m_{1} C_{M}\left(a_{1}\right) \subseteq C_{M}\left(a_{2}\right) \cup \cdots \cup C_{M}\left(a_{s}\right),
$$

since the right-hand side is $b$-invariant. Then

$$
M=C_{M}\left(a_{2}\right) \cup \cdots \cup C_{M}\left(a_{s}\right) .
$$

If $b$ normalizes the coset $y^{-1} m_{1} C_{M}\left(a_{1}\right)$, then, since the action of $b$ on $M$ is coprime, it follows that $y^{-1} m_{1} C_{M}\left(a_{1}\right)$ contains an element $m_{0} \in C_{M}(b)=\bigcap_{i=2}^{s} C_{M}\left(a_{i}\right)$. For any $x \in$ 
$m_{0} C_{M}\left(a_{1}\right)=y^{-1} m_{1} C_{M}\left(a_{1}\right) \neq C_{M}\left(a_{1}\right)$ the element $m_{0} x$ is not in the coset $y^{-1} m_{1} C_{M}\left(a_{1}\right)$ and therefore $m_{0} x \in C_{M}\left(a_{i}\right)$ for some $i \geqslant 2$ by (4.2). Then $x \in m_{0}^{-1} C_{M}\left(a_{i}\right)=C_{M}\left(a_{i}\right)$, so that again

and by (4.2)

$$
y^{-1} m_{1} C_{M}\left(a_{1}\right) \subseteq C_{M}\left(a_{2}\right) \cup \cdots \cup C_{M}\left(a_{s}\right)
$$

$$
M=C_{M}\left(a_{2}\right) \cup \cdots \cup C_{M}\left(a_{s}\right) .
$$

As a result of (4.1),(4.3),(4.4), in all cases,

$$
M=C_{M}\left(a_{1}\right) \cup C_{M}\left(a_{2}\right) \cup \cdots \cup C_{M}\left(a_{s}\right) .
$$

Note also that the automorphism $a_{1} a_{2} \cdots a_{s}$ acts faithfully on $M$, as $M \neq C_{M}\left(a_{i}\right)$ for every $i$. This situation is known to be impossible due to the following well-known lemma, which we prove here for completeness.

Lemma 4.6. If $\alpha$ is an automorphism of an elementary abelian q-group $V$, then there is an element $v \in V$ such that $C_{\langle\alpha\rangle}(v)=1$.

Proof. Induction on $|V|$. If $|V|=q$, then every element in $\langle\alpha\rangle$ acts without non-trivial fixed points, and the result follows. In the general case, if an element $\beta \in\langle\alpha\rangle$ of prime order $r \neq q$ has non-trivial fixed points, then $V=C_{V}(\beta) \times[V, \beta]$ by Maschke's theorem, and both factors are $\alpha$-invariant and have smaller order than $V$. By induction, there are $v_{1} \in C_{V}(\beta)$ and $v_{2} \in[V, \beta]$ such that $C_{\langle\alpha\rangle}\left(v_{1}\right)=C_{\langle\alpha\rangle}\left(C_{V}(\beta)\right)$ and $C_{\langle\alpha\rangle}\left(v_{2}\right)=C_{\langle\alpha\rangle}([V, \beta])$. Then $C_{\langle\alpha\rangle}\left(v_{1}+v_{2}\right)=C_{\langle\alpha\rangle}\left(C_{V}(\beta)\right) \cap C_{\langle\alpha\rangle}([V, \beta])=C_{\langle\alpha\rangle}(V)=1$.

Thus, we can assume that all elements of $\langle\alpha\rangle$ of order coprime to $q$ act on $V$ without nontrivial fixed points. It remains to choose an element of $V$ outside the centralizer of an element of $\langle\alpha\rangle$ of order $q$ (or any non-trivial element of $V$ if $q \nmid|\alpha|$ ).

The proof of Proposition 4.5 is complete.

\section{Nonsoluble LENGTH}

In this section we prove Theorem 1.3. First we introduce some notation. Consider the 'upper nonsoluble series' of $G$, which by definition starts from the soluble radical $R_{0}=R(G)$ and the full inverse image $L_{1}$ of $F^{*}\left(G / R_{0}\right)$. Then by induction $R_{j}$ is the full inverse image of the soluble radical of $G / L_{j}$, and $L_{j+1}$ the full inverse image of $F^{*}\left(G / R_{j}\right)$. It is easy to see that the nonsoluble length $\lambda(G)$ is equal to the first positive integer $l$ such that $R_{l}=G$. In the normal series

$$
1=L_{0} \leqslant R_{0}<L_{1} \leqslant R_{1}<\cdots \leqslant R_{l}=G
$$

each quotient $U_{i}=L_{i} / R_{i-1}$ is a (nontrivial) direct product of nonabelian simple groups, and each quotient $R_{i} / L_{i}$ is soluble (possibly trivial). By the well-known properties of the generalized Fitting subgroup, if we write one of those nonsoluble quotients as a direct product $U_{j}=S_{1} \times \cdots \times S_{v}$ of nonabelian simple groups $S_{i}$, then the set of these factors $S_{i}$ is uniquely determined as the set of subnormal simple subgroups of $G / R_{j-1}$. Acting by conjugation the group $G$ permutes these subnormal factors; for brevity we simply speak of orbits of elements of $G$ on $U_{j}$ meaning orbits in this permutational action. The stabilizer of a point $S_{i}$ can also be denoted as the normalizer $N_{G}\left(S_{i}\right)$ of the section $S_{i}$.

The subgroup $L_{j} / R_{j-1}$ contains its centralizer in $G / R_{j-1}$. Let $K_{j}$ be the kernel of the permutational action of $G$ on $\left\{S_{1}, \ldots, S_{v}\right\}$. Clearly, $L_{j} \leqslant K_{j}$. The quotient $K_{j} / L_{j}$ is soluble by the Schreier conjecture. Therefore, $K_{j} \leqslant R_{j}$. We shall routinely use these facts without special references. 
Let $g$ be an element of a finite group $G$, and let $\left\{S_{1}, \ldots, S_{r}\right\}$ be a $g$-orbit in $U_{i}$. We say that the orbit is pure if the order of the automorphism of $S=S_{1} \cdots S_{r}$ induced by $g$ acting by conjugation is equal to $r$, which is the order of the permutation induced by $g$ on this orbit; in other words, if the stabilizer of a point in $\langle g\rangle$ acts trivially on $S$ : $N_{\langle g\rangle}\left(S_{1}\right)=C_{\langle g\rangle}(S)$.

We now prove two key technical propositions. It is convenient to introduce the following hypothesis.

Hypothesis 5.1. Let $g$ be an element of a finite group $G$, and let $\left\{S_{1}, \ldots, S_{r}\right\}$ be a g-orbit in a section $U_{i}=L_{i} / R_{i-1}$ of the series (5.1) with $i \geqslant 2$. Suppose that $\langle g\rangle \cap L_{i}=1$, and that $g$ acting by conjugation induces a nontrivial automorphism $\bar{g}$ of $S=S_{1} \cdots S_{r}$. Let $t=|\bar{g}|$, so that $\left\langle g^{t}\right\rangle$ is the centralizer of $S$ in $\langle g\rangle$ and $\langle\bar{g}\rangle=\langle g\rangle /\left\langle g^{t}\right\rangle$.

Furthermore, let $\hat{S}$ be a minimal by inclusion g-invariant subgroup of $G$ such that $\hat{S} R_{i-1} / R_{i-1}=S$. If in addition $\left\{S_{1}, \ldots, S_{r}\right\}$ is a pure $g$-orbit, then we choose $\hat{S}$ to be also contained in $E_{n}(g)$, which is possible by Lemma 3.9. Let $\hat{S}_{1}$ be a minimal by inclusion subgroup of $\hat{S}$ such that $\hat{S}_{1} R_{i-1} / R_{i-1}=S_{1}$.

The first of the technical propositions provides a passage from a pure orbit of $g$ in $U_{i}$ to an orbit of at least the same length in the preceding section $U_{i-1}$.

Proposition 5.2. Assume Hypothesis 5.1. If $\left\{S_{1}, \ldots, S_{r}\right\}$ is a pure g-orbit, then $U_{i-1}$ contains a g-orbit $\left\{T_{1}, \ldots, T_{l}\right\}$ of length divisible by $|\bar{g}|$ and for some choice of $\hat{S}_{1}$ there is an element $x_{0} \in \hat{S}_{1} \backslash R_{i-1}$ that does not belong to $N_{G}\left(T_{1}\right)$.

Proof. Consider the permutational action of the group $\hat{S}\langle g\rangle$ on the set of simple factors of $U_{i-1}$. Since the kernel of this action is contained in $R_{i-1}$, for an element $x_{0} \in \hat{S}_{1} \backslash R_{i-1}$ there is an orbit $\left\{T_{1}, \ldots, T_{s}\right\}$ such that the kernel $K$ of the restriction to this orbit does not contain $x_{0}$. In particular, $\hat{S} \nless K$. Then $K$ cannot cover $S$ by minimality of $\hat{S}$. Since $K$ is a normal subgroup of $\hat{S}\langle g\rangle$, it follows that $K \leqslant\left(\hat{S} \cap R_{i-1}\right)\left\langle g^{t}\right\rangle$ (where, recall, $\left\langle g^{t}\right\rangle$ is the centralizer of $S$ in $\langle g\rangle)$. In particular, $K \leqslant C_{\hat{S}\langle g\rangle}(S)$.

Let $W=\hat{S} K / K$ and let $R$ be the soluble radical of $W$; then $W / R \cong S$ since $L_{j-1} \leqslant K$. Let $\tilde{g}$ be the image of $g$ in $\hat{S}\langle g\rangle / K$. We claim that $C_{\langle\tilde{g}\rangle}(S)=\left\langle\tilde{g}^{t}\right\rangle$. Indeed, if $\left[\hat{S}, g^{i}\right] \leqslant$ $\left(R_{i-1} \cap \hat{S}\right) K \leqslant R_{i-1}\left\langle g^{t}\right\rangle$, then $\left[\left[\hat{S}, g^{i}\right], g^{i}\right] \leqslant R_{i-1}$, whence $\left[\hat{S}, g^{i}\right] \leqslant R_{i-1}$, since then the image of $g^{i}$ in $\hat{S}\langle g\rangle /\left(R_{i-1} \cap \hat{S}\right)$ belongs to the Fitting subgroup by Baer's theorem.

We consider the action of $W\langle\tilde{g}\rangle$ on the orbit $\left\{T_{1}, \ldots, T_{s}\right\}$. Let $\tilde{H}$ be the stabilizer of $T_{1}$ in $W\langle\tilde{g}\rangle$. Then $W \nless \tilde{H}$, since $\hat{S} \nless K$. We now show that the triple $W, \tilde{g}, \tilde{H}$ satisfies the other hypotheses of Proposition 4.5. Condition (1): if $\tilde{M} \leqslant W$ for a $g$-invariant subgroup $\tilde{M}$ covering $S$, then for the full inverse image $M$ we have $M \leqslant \hat{S} K \leqslant \hat{S}\left\langle g^{t}\right\rangle$. Then $[M, M] \leqslant\left[\hat{S}\left\langle g^{t}\right\rangle, \hat{S}\left\langle g^{t}\right\rangle\right] \leqslant \hat{S}$. Since $[M, M]$ also covers $S$, by minimality of $\hat{S}$ we must have $[M, M]=\hat{S}$, whence $\tilde{M}=W$. Condition (2): if $a k=g^{i}$ for $a \in \hat{S}, k \in K$, then $b=g^{i} g^{t j}$ for some $b \in \hat{S}$ and some $j$, because $K \leqslant\left(\hat{S} \cap R_{i-1}\right)\left\langle g^{t}\right\rangle$. Since $\langle g\rangle \cap \hat{S}=1$ by Hypothesis 5.1 , we obtain $b=g^{i} g^{t j}=1$, so that $g^{i} \in\left\langle g^{t}\right\rangle$ and $\tilde{g}^{i} \in C_{\langle\tilde{g}\rangle}(S)$. Conditions (3) and (4) clearly hold.

By Proposition 4.5 there is $\tilde{w} \in W$ such that

$$
\tilde{H}^{\tilde{w}} \cap\langle\tilde{g}\rangle \leqslant\left\langle\tilde{g}^{t}\right\rangle \text {. }
$$

Let $H$ be the full inverse image of $\tilde{H}$ in $\hat{S}\langle g\rangle$, and $w$ some pre-image of $\tilde{w}$. Then

$$
H^{w} \cap\langle g\rangle \leqslant\left\langle g^{t}\right\rangle K \cap\langle g\rangle \leqslant C_{\hat{S}\langle g\rangle}(S) \cap\langle g\rangle=\left\langle g^{t}\right\rangle .
$$


Since $H$ is the stabilizer of $T_{1}$ in $\hat{S}\langle g\rangle$, this means that the $g$-orbit in $U_{i-1}$ containing $T_{1}^{w}$ has length divisible by $t=|\bar{g}|$.

Recall that $x_{0} \in \hat{S}_{1} \backslash R_{i-1}$ and $x_{0} \notin K$. There is $y \in \hat{S}\langle g\rangle$ such that $x_{0} \notin H^{w y}$. Since $y=g^{j} s$ for some integer $j$ and some $s \in \hat{S}$, we have $x_{0} \notin H^{w y}=H^{w g^{j} s}$, so that $x_{0}^{s^{-1}} \notin H^{w g^{j}}$. Thus the element $x_{0}^{s^{-1}}$, which belongs to $\hat{S}_{1}^{s^{-1}} \backslash R_{i-1}$, moves $T_{1}^{w g^{j}}$. Note that $\hat{S}_{1}^{s^{-1}}$ is also a minimal subgroup of $\hat{S}$ covering $S_{1}$. Clearly, the $g$-orbit containing $T_{1}^{w g^{j}}$ has the same length as the $g$-orbit containing $T_{1}^{w}$, and this length divides $|\bar{g}|$ by (5.2). It remains to rename $T_{1}^{w g^{j}}$ by $T_{1}, \hat{S}_{1}^{s^{-1}}$ by $\hat{S}_{1}$, and $x_{0}^{s^{-1}}$ by $x_{0}$. The proposition is proved.

The next technical proposition provides a passage from a non-pure orbit of $g$ in $U_{i}$ to an orbit in $U_{i-1}$ that is strictly greater with respect to the following ordering. Namely, on the set of positive integers we introduce the lexicographical order with respect to the exponents of primes in the canonical prime-power decomposition: if

$$
a=2^{k_{2}} \cdot 3^{k_{3}} \cdot 5^{k_{5}} \cdot 7^{k_{7}} \ldots \quad \text { and } \quad b=2^{l_{2}} \cdot 3^{l_{3}} \cdot 5^{l_{5}} \cdot 7^{l_{7}} \ldots,
$$

then by definition $a \prec b$ if, for some prime $p$, we have $k_{q}=l_{q}$ for all primes $q<p$ and $k_{p}<l_{p}$. Clearly, if $u$ is divisible by $v$, then $v \prec u$.

Proposition 5.3. Assume Hypothesis 5.1. If $\left\{S_{1}, \ldots, S_{r}\right\}$ is a non-pure g-orbit, then $U_{i-1}$ contains a g-orbit $\left\{T_{1}, \ldots, T_{l}\right\}$ of length strictly greater than $r$ with respect to the order $\prec$.

Proof. Let $|\bar{g}|=p_{1}^{\alpha_{1}} \cdot p_{2}^{\alpha_{2}} \cdots$ for primes $p_{1}<p_{2}<\cdots$ and positive integers $\alpha_{i}$. Let $\bar{g}_{i}$ be a generator of the Sylow $p_{i}$-subgroup of $\langle\bar{g}\rangle$, and let $g_{i}$ be an inverse image of $\bar{g}_{i}$ in the Sylow $p_{i}$-subgroup of $\langle g\rangle$.

Since the orbit is non-pure, the stabilizer of a point $S_{1}$ in the permutational action of $\bar{g}$ on $\left\{S_{1}, \ldots, S_{r}\right\}$ is nontrivial. Let the order of this stabilizer be $p_{1}^{\beta_{1}} \cdot p_{2}^{\beta_{2}} \cdots$ for the same primes $p_{i}$ and non-negative integers $\beta_{i}$, not all of which are zero. Let $j$ be the smallest index such that $\beta_{j} \geqslant 1$. Consider the element

$$
f=g_{1} \cdots g_{j-1} \cdot g_{j}
$$

(which generates the Hall $\left\{p_{1}, \ldots, p_{j}\right\}$-subgroup of $\langle g\rangle$ ). Here, the case $j=1$ is not excluded, when $f=g_{1}$. Let $\bar{f}$ be the image of $f$ in $\langle g\rangle /\left\langle g^{t}\right\rangle$, so that

$$
|\bar{f}|=p_{1}^{\alpha_{1}} \cdots p_{j}^{\alpha_{j}} .
$$

Let $t_{0}=|\bar{f}|$ for brevity.

Consider an orbit of $f$ in $\left\{S_{1}, \ldots, S_{r}\right\}$, which we denote by $\left\{V_{1}, \ldots, V_{v}\right\}$. Let $V=$ $V_{1} \cdots V_{v}$, and let $\hat{V}$ be a minimal $f$-invariant subgroup of $\hat{S}$ such that $\hat{V} R_{i-1} / R_{i-1}=V$. Let $\check{f}$ be the automorphism of $V$ induced by $f$. Since the products of simple factors over $f$-orbits are permuted by $g$, the centralizer of $V$ in $\langle f\rangle$ is equal to $\langle f\rangle \cap\left\langle g^{t}\right\rangle=\left\langle f^{t_{0}}\right\rangle$ (recall that $\left\langle g^{t}\right\rangle$ is the centralizer of $S$ in $\left.\langle g\rangle\right)$. Hence,

$$
|\check{f}|=|\bar{f}|=p_{1}^{\alpha_{1}} \cdots p_{j-1}^{\alpha_{j-1}} \cdot p_{j}^{\alpha_{j}} .
$$

Note that by construction the stabilizer of a point $V_{1}$ in $\langle\check{f}\rangle$ is a $p_{j}$-subgroup.

Consider the permutational action of the group $\hat{V}\langle f\rangle$ on the set of simple factors of $U_{i-1}$. Since the kernel of this action is contained in $R_{i-1}$, we can choose an orbit $\left\{T_{1}, \ldots, T_{l}\right\}$ for which the kernel $K$ of the restriction to this orbit does not contain some element $x \in \hat{V}_{1} \backslash R_{i-1}$. Then $K$ cannot cover $V$ by minimality of $\hat{V}$. Since $K$ is a normal subgroup of $\hat{V}\langle f\rangle$, it follows that $K \leqslant\left(\hat{V} \cap R_{i-1}\right)\left\langle f^{t_{0}}\right\rangle$. In particular, $K \leqslant C_{\hat{V}\langle f\rangle}(V)$. 
Let $W=\hat{V} K / K$ and let $R$ be the soluble radical of $W$; then $W / R \cong V$, since $L_{j-1} \leqslant K$. Let $\tilde{f}$ be the image of $f$ in $\hat{V}\langle f\rangle / K$. We claim that $C_{\langle\tilde{f}\rangle}(V)=\left\langle\tilde{f}^{t_{0}}\right\rangle$. Indeed, if $\left[\hat{V}, f^{i}\right] \leqslant$ $\left(R_{i-1} \cap \hat{V}\right) K \leqslant R_{i-1}\left\langle g^{t}\right\rangle$, then $\left[\left[\hat{V}, f^{i}\right], f^{i}\right] \leqslant R_{i-1}$, whence $\left[\hat{V}, f^{i}\right] \leqslant R_{i-1}$, since then the image of $f^{i}$ in $\hat{V}\langle f\rangle /\left(R_{i-1} \cap \hat{V}\right)$ belongs to the Fitting subgroup by Baer's theorem.

We consider the action of $W\langle\tilde{f}\rangle$ on the orbit $\left\{T_{1}, \ldots, T_{l}\right\}$. Let $\tilde{H}$ be the stabilizer of $T_{1}$ in $W\langle\tilde{f}\rangle$. Then $W \nless \tilde{H}$, since $\hat{V} \not K$.

We now show that the triple $W, \tilde{f}, \tilde{H}$ satisfies the other hypotheses of Proposition 4.5 in the role of $G, g, H$. Condition (1): if $\tilde{M} \leqslant W$ for an $f$-invariant subgroup $\tilde{M}$ covering $V$, then for the full inverse image $M$ we have $M \leqslant \hat{V} K \leqslant \hat{S}\left\langle f^{t_{0}}\right\rangle$. Then $[M, M] \leqslant$ $\left[\hat{V}\left\langle f^{t_{0}}\right\rangle, \hat{V}\left\langle f^{t_{0}}\right\rangle\right] \leqslant \hat{V}$. Since $[M, M]$ also covers $V$, by minimality of $\hat{V}$ we must have $[M, M]=\hat{V}$, whence $\tilde{M}=W$. Condition (2): if $a k=f^{i}$ for $a \in \hat{V}, k \in K$, then $b=f^{i} f^{t_{0} j}$ for some $b \in \hat{V}$ and some $j$, because $K \leqslant\left(\hat{V} \cap R_{i-1}\right)\left\langle f^{t_{0}}\right\rangle$. Since $\langle f\rangle \cap \hat{V}=1$ by Hypothesis 5.1, we obtain $b=f^{i} f^{t_{0} j}=1$, so that $f^{i} \in\left\langle f^{t_{0}}\right\rangle$ and $\tilde{f}^{i} \in C_{\langle\tilde{f}\rangle}(V)$. Conditions (3) and (4) clearly hold by construction.

By Proposition 4.5 there is $\tilde{w} \in W$ such that

$$
\tilde{H}^{\tilde{w}} \cap\langle\tilde{f}\rangle \leqslant\left\langle\tilde{f}^{t_{0}}\right\rangle .
$$

Let $H$ be the full inverse image of $\tilde{H}$ in $\hat{V}\langle f\rangle$, and $w$ some pre-image of $\tilde{w}$. Then

$$
H^{w} \cap\langle f\rangle \leqslant\left\langle f^{t_{0}}\right\rangle K \cap\langle f\rangle \leqslant C_{\hat{V}\langle f\rangle}(V) \cap\langle f\rangle=\left\langle f^{t_{0}}\right\rangle .
$$

Since $H$ is the stabilizer of $T_{1}$ in $\hat{V}\langle f\rangle$, this means that the $f$-orbit in $U_{i-1}$ containing $T_{1}^{w}$ has length divisible by

$$
|\check{f}|=p_{1}^{\alpha_{1}} \cdots p_{j-1}^{\alpha_{j-1}} \cdot p_{j}^{\alpha_{j}} .
$$

Then the $g$-orbit containing $T_{1}^{w}$ also has length divisible by

$$
p_{1}^{\alpha_{1}} \cdots p_{j-1}^{\alpha_{j-1}} \cdot p_{j}^{\alpha_{j}} .
$$

This number is strictly greater with respect to our lexicographical order $\prec$ than the length of the original orbit

$$
p_{1}^{\alpha_{1}} \cdots p_{j-1}^{\alpha_{j-1}} \cdot p_{j}^{\alpha_{j}-\beta_{j}} \cdots
$$

by the choice of $j$. It remains to rename $T_{1}^{w}$ by $T_{1}$. The proposition is proved.

Theorem 1.3 will follow by induction from the following proposition. Recall that $K_{i}$ is the kernel of the permutational action of $G$ on the set of simple factors of $U_{i}=L_{i} / R_{i-1}$.

Proposition 5.4. Let $g$ be an element of a finite group $G$ whose order $|g|$ is equal to the product of $m$ primes counting multiplicities. Suppose that $\langle g\rangle \cap K_{m s}=1$ for some positive integer $s$. Then for any positive integer $n$ the nonsoluble length of $E_{n}(g)$ is at least $s$.

Proof. Consider the 'upper nonsoluble series' for $E_{n}(g)$ constructed in the same way as (5.1) was constructed for $G$, with its terms denoted by

$$
1=\lambda_{0} \leqslant \rho_{0}<\lambda_{1} \leqslant \rho_{1}<\cdots \leqslant \rho_{e}=E_{n}(a),
$$

where $e=\lambda\left(E_{n}(g)\right)$ is the nonsoluble length of $E_{n}(g)$. Namely, $\rho_{0}=R\left(E_{n}(g)\right)$ is the soluble radical, $\lambda_{1}$ is the full inverse image of $F^{*}\left(E_{n}(g) / \rho_{0}\right)$, and by induction $\rho_{j}$ is the full inverse image of the soluble radical of $E_{n}(g) / \lambda_{j}$, and $\lambda_{j+1}$ the full inverse image of $F^{*}\left(E_{n}(g) / \rho_{j}\right)$. The quotients $\lambda_{i} / \rho_{i-1}$ are (nontrivial) direct products of nonabelian simple groups, and the quotients $\rho_{i} / \lambda_{i}$ (possibly trivial) are soluble. Thus, $e$ is the first positive integer such that $\rho_{e}=E_{n}(g)$. Our task is to show that $e \geqslant s$. 
Since $\langle g\rangle \cap K_{m s}=1$, the element $g$ has at least one nontrivial orbit on the set of simple factors of $U_{m s}=L_{m s} / R_{m s-1}$, say, $\left\{S_{1}, \ldots, S_{r}\right\}$. If the orbit is pure, then we apply Proposition 5.2 to this orbit. If the orbit is not pure, then we apply Proposition 5.3. Then we apply the same procedure to the orbit $\left\{T_{1}, \ldots, T_{l}\right\}$ in $U_{m s-1}$ thus obtained: this orbit takes the role of the orbit $\left\{S_{1}, \ldots, S_{r}\right\}$ in Proposition 5.2 or 5.3 depending on whether it is pure or not. We proceed with constructing this sequence of orbits, descending over the sections $U_{i}$ making $m s-1$ such steps. If we make such a step from a pure orbit by Proposition 5.2, then the length of the new orbit is divisible by the length of the old orbit and therefore does not decrease with respect to the order $\prec$. If we make such a step from a non-pure orbit by Proposition 5.3, then the length of the new orbit is strictly greater than the length of the old orbit with respect to the order $\prec$.

In the sequence of orbits thus constructed, some orbits may be pure, some not. We can visualize this fact as a sequence of $P$ s (for pure) and $N$ s (for non-pure), like

$$
P \rightarrow N \rightarrow P \rightarrow P \rightarrow P \rightarrow N \rightarrow N \rightarrow P \rightarrow N \rightarrow P \rightarrow \cdots
$$

Importantly, in every passage of types $N \rightarrow N$ or $N \rightarrow P$ the length of the orbit strictly increases with respect to the order $\prec$, while at passages of types $P \rightarrow N$ or $P \rightarrow P$ the length of the orbit does not decrease with respect to the order $\prec$. Therefore there can be at most $m-1$ passages of type $N \rightarrow N$ or $N \rightarrow P$, that is, at most $m-1$ occurrences of $N$ in this sequence. As a result, if the length of the sequence is at least $(s-1) m+(m-1)+1=m s$, then it will necessarily contain a subsequence

$$
P \rightarrow P \rightarrow \cdots \rightarrow P
$$

of $s$ pure orbits with $s-1$ consecutive passages $P \rightarrow P$. Let $i=t, t-1, \ldots, t-s+1$ be the indices of the corresponding sections $U_{i}$.

Recall that if a $g$-orbit $\left\{S_{1}, \ldots, S_{r}\right\}$ in $U_{i}$ is pure, then by Lemma 3.9 the subgroup $S=S_{1} \times \cdots \times S_{r}$ is contained in the image of $E_{n}(g)$ in $G / R_{i-1}$ (since this image is obviously equal to the analogous subgroup $E_{n}(g)$ constructed for $\left.G / R_{i-1}\right)$.

The idea is to use each of these $s$ consecutive pure orbits in $U_{t}, U_{t-1}, \ldots, U_{t-s+1}$ to 'mark' a nonsoluble factor of the series (5.3) and prove that the factor 'marked' by the pure orbit in $U_{i-1}$ is necessarily 'lower' in (5.3) than the factor marked by the pure orbit in $U_{i}$, for every $i=t, t-1, \ldots, t-s+2$. Then the series (5.3) must realize nonsoluble length at least $s$.

Thus, let $\left\{S_{1}, \ldots, S_{r}\right\}$ be a pure $g$-orbit in $U_{i}$, and $\left\{T_{1}, \ldots, T_{l}\right\}$ the pure $g$-orbit in $U_{i-1}$ obtained by Proposition 5.2. Recall that in accordance with Hypothesis 5.1, $\hat{S}$ is a minimal by inclusion $g$-invariant subgroup of $E_{n}(g)$ such that $S=\hat{S} R_{i-1} / R_{i-1}$, and $\hat{S}_{1}$ is a minimal by inclusion subgroup of $\hat{S}$ such that $\hat{S}_{1} R_{i-1} / R_{i-1}=S_{1}$. Note that since $S_{1}$ is nonabelian simple, $\hat{S}_{1}$ has no nontrivial soluble homomorphic images:

$$
\hat{S}_{1}=\left[\hat{S}_{1}, \hat{S}_{1}\right]
$$

Recall also that by Proposition 5.2 we have an element $x_{0} \in \hat{S}_{1} \backslash R_{i-1}$ such that

$$
T_{1}^{x_{0}} \neq T_{1}
$$

Consider the image of the series (5.3) in $E_{n}(g) R_{i-1} / R_{i-1}$. Since $\hat{S}_{1} R_{i-1} / R_{i-1} \cong S_{1}$ is a subnormal nonabelian simple subgroup of $E_{n}(g) R_{i-1} / R_{i-1}$, we obviously have a welldefined index $j$ such that

$$
\hat{S}_{1} \leqslant \lambda_{j} R_{i-1} \quad \text { and } \quad \hat{S}_{16} \not \rho_{j-1} R_{i-1} .
$$


Note that then also

$$
\hat{S}_{1} \leqslant \lambda_{j}\left(R_{i-1} \cap E_{n}(g)\right) .
$$

It is also clear that the index $j$ depends only on $S_{1}$ (that is, it is independent of the choice of $\hat{S}_{1}$ such that $\hat{S}_{1} R_{i-1} / R_{i-1}=S_{1}$ ).

Since $\left\{T_{1}, \ldots, T_{l}\right\}$ is also a pure $g$-orbit in $U_{i-1}$, the product $T=T_{1} \cdots T_{l}$ is also covered by $E_{n}(g)$ by Lemma 3.9 . If $\hat{T}_{1}$ is any subgroup of $E_{n}(g)$ such that $\hat{T}_{1} R_{i-2} / R_{i-2}=T_{1}$, then, again, there is a well-defined index $u$ depending only on $T_{1}$ such that

$$
\hat{T}_{1} \leqslant \lambda_{u}\left(R_{i-2} \cap E_{n}(g)\right) \quad \text { and } \quad \hat{T}_{1} \nless \rho_{u-1} R_{i-2} .
$$

Lemma 5.5. Under the above hypotheses, $j>u$.

Proof. We argue by contradiction. Suppose that $j \leqslant u$. Then (5.7) implies that $\hat{S}_{1} \leqslant$ $\lambda_{u}\left(R_{i-1} \cap E_{n}(g)\right)$. The image of $\lambda_{u} / \rho_{u-1}$ in $E_{n}(g) /\left(R_{i-2} \cap E_{n}(g)\right.$ is a direct product of nonabelian simple groups, one of which is $\bar{T}_{1}=\hat{T}_{1} /\left(\hat{T}_{1} \cap R_{i-2}\right) \cong T_{1}$ by (5.8). Acting by conjugation the group $E_{n}(g)$ permutes these factors. Consider the permutational action of $E_{n}(g)$ on the orbit containing $\bar{T}_{1}$. Clearly, $\lambda_{u}$ is contained in the kernel of this action. The subgroup $L_{i-1} \cap E_{n}(g)$ normalizes $\hat{T}_{1}$ modulo $R_{i-2} \cap E_{n}(g)$ (since $L_{i-1}$ normalizes $\hat{T}_{1}$ modulo $\left.R_{i-2}\right)$. As a normal subgroup of $E_{n}(g)$, then $L_{i-1} \cap E_{n}(g)$ is contained in the kernel of that action on the orbit containing $\bar{T}_{1}$. Since $R_{i-1} / L_{i-1}$ is soluble, we obtain that the image of $\hat{S}_{1} \leqslant \lambda_{u}\left(R_{i-1} \cap E_{n}(g)\right)$ in this action is soluble. But $\hat{S}_{1}=\left[\hat{S}_{1}, \hat{S}_{1}\right]$ by the minimality of $\hat{S}_{1}$, as noted in (5.4). Therefore this image must actually be trivial; in particular,

$$
\hat{T}_{1}^{x_{0}} \equiv \hat{T}_{1}\left(\bmod \rho_{u-1}\left(R_{i-2} \cap E_{n}(g)\right)\right) .
$$

On the other hand, since $T_{1}^{x_{0}} \neq T_{1}$ by (5.5), it follows that $\left[\hat{T}_{1}, \hat{T}_{1}^{x_{0}}\right] \leqslant R_{i-2} \cap E_{n}(g)$. Together with (5.9) this implies

$$
\bar{T}_{1}=\left[\bar{T}_{1}, \bar{T}_{1}\right] \equiv 1\left(\bmod \rho_{u-1}\left(R_{i-2} \cap E_{n}(g)\right)\right),
$$

contrary to $(5.8)$.

We now finish the proof of Proposition 5.4. Each pure orbit $\left\{S_{1}, \ldots, S_{r}\right\}$ in $U_{i}$ for $i=t, \ldots, t-s+1$ in our sequence constructed by successive application of Propositions 5.2 or 5.3 marks a nonsoluble quotient $\lambda_{j} / \rho_{j-1}$ of the series (5.3) in the sense of (5.6). By Lemma 5.5 the next pure orbit in $U_{i-1}$ marks a strictly lower section. Therefore there must be at least $s$ different nonsoluble sections in (5.3), since their indices must be strictly descending as we go over the $s$ consecutive pure orbits. As a result, the nonsoluble length of $E_{n}(g)$ is at least $s$.

Proof of Theorem 1.3. Recall that we have $\lambda\left(E_{n}(g)\right)=k$ and $|g|$ is a product of $m$ primes counting multiplicities; we need to show that $g \in R_{(k+1) m(m+1) / 2}(G)$. By Proposition 5.4 we have

$$
\langle g\rangle \cap R_{(k+1) m} \geqslant\langle g\rangle \cap K_{(k+1) m} \neq 1 .
$$

The similar subgroup $E_{n}(\bar{g})$ constructed for the image $\bar{g}$ of $g$ in $\bar{G}=G / R_{(k+1) m}$ is clearly the image of $E_{n}(g)$ and therefore its nonsoluble length is at most $k$. Then $\bar{g} \in R_{(k+1)(m-1) m / 2}(\bar{G})$ by induction on $m$. The result follows, since $R_{(k+1)(m-1) m / 2}(\bar{G})$ is the image of $R_{(k+1) m(m+1) / 2}$ in $G / R_{(k+1) m}$. 


\section{Generalized Fitting height}

Proof of Theorem 1.2. Recall that $g$ is an element of a finite group $G$ whose order $|g|$ is equal to the product of $m$ primes counting multiplicities, and the generalized Fitting height of $E_{n}(g)$ is equal to $k$. We need to show $g$ belongs to $F_{((k+1) m(m+1)+2)(k+3) / 2}^{*}(G)$.

As in the proof of Theorem 1.1, consider the subnormal $g$-invariant subgroups

$$
[\ldots[[G, g], g], \ldots, g] \text {, }
$$

formed by taking successive commutator subgroups. Let $H$ be the smallest of the subgroups $[\ldots[[G, g], g], \ldots, g]$. Note that if $H=1$, then $g$ is a left-Engel element and therefore $g \in F(G)$ and there is nothing to prove. In any case, $H=[H, g]$.

Let $N=\left\langle H^{G}\right\rangle$ be the normal closure of $H$. By construction, the image of $g$ in $G / N$ is a left-Engel element and therefore belongs to the Fitting subgroup of $G / N$. If we prove that the generalized Fitting height of $N$ is at most $h$ for some number $h$, then $N$, being a normal subgroup, is contained in $F_{h}^{*}(G)$ and then $g \in F_{h+1}^{*}(G)$. Since $H$ is subnormal in $G$, by Lemma 2.1(b) the generalized Fitting height of its normal closure $N$ is the same as that of $H$. Therefore it suffices to obtain the appropriate estimate of the generalized Fitting height of $H$.

Consider the group $H\langle g\rangle$. Since the nonsoluble length of $E_{n}(g)$ does not exceed its generalized Fitting height, by Theorem 1.3 the element $g$ belongs to $R_{(k+1) m(m+1) / 2}(G)$. Since $H=[H, g]$, it follows that $H \leqslant R_{(k+1) m(m+1) / 2}(G)$, and since $H$ is a subnormal subgroup, $\lambda(H) \leqslant(k+1) m(m+1) / 2$ by Lemma $2.1($ a). It remains to obtain bounds for the Fitting height of the $(k+1) m(m+1) / 2+1$ soluble factors of the upper nonsoluble series of $H$, which are $g$-invariant together with $H$.

Let $X=Y / Z$ be one of such soluble factors, where $Y$ and $Z$ are $g$-invariant and normal in $H$ and therefore subnormal in $G$. Since $Y$ is subnormal, we have $h^{*}\left(E_{n}(g) \cap Y\right) \leqslant$ $h^{*}\left(E_{n}(g)\right)=k$ by Lemma 2.1(a). Then the (soluble) image of $E_{n}(g) \cap Y$ in $Y / Z$ has Fitting height at most $k$. Since, clearly, $E_{Y, n}(g) \leqslant E_{n}(g) \cap Y$, we obtain that $E_{X, n}(g)$, which is the image of $E_{Y, n}(g)$, also has Fitting height at most $k$.

Consider the 'outer' semidirect product $X \rtimes\langle g\rangle$ (recall that both $Y$ and $Z$ are $g$ invariant, so this semidirect product is well defined). By applying Theorem 1.1 we obtain that $g \in F_{k+1}(X \rtimes\langle g\rangle)$. Therefore $[X, g] \leqslant F_{k+1}(X \rtimes\langle g\rangle) \cap X \leqslant F_{k+1}(X)$. In other words, $g$ acts trivially on $X / F_{k+1}(X)$. Since $H=[H, g]$, it follows that $H$ also acts trivially on $X / F_{k+1}(X)$, that is, $X / F_{k+1}(X)$ is a central section of $H$. In particular, $X=F_{k+2}(X)$, that is, the Fitting height of $X$ is at most $k+2$.

Thus, the generalized Fitting height of $H$ is at most $((k+1) m(m+1) / 2+1)(k+2)+$ $(k+1) m(m+1) / 2=((k+1) m(m+1)+2)(k+3) / 2-1$, as required.

\section{FinAl REMARKS AND CONJECTURES}

We conjecture that the results of Theorems 1.2 and 1.3 can be strengthened by removing the dependence on the order of the element. In fact, we have quite precise conjectures, with best-possible values. For generalized Fitting height we state the following.

Conjecture 7.1. Let $g$ be an element of a finite group $G$, and $n$ a positive integer. If the generalized Fitting height of $E_{n}(g)$ is equal to $k$, then $g \in F_{k+1}^{*}(G)$.

For nonsoluble length we state the following.

Conjecture 7.2. Let $g$ be an element of a finite group $G$, and $n$ a positive integer. If the nonsoluble length of $E_{n}(g)$ is equal to $k$, then $g \in R_{k}(G)$. 
As we show below, it is in this strongest form that Conjectures 7.1 and 7.2 can be derived from an affirmative answer to the following question about automorphisms of direct products of nonabelian finite simple groups.

Question 7.3. Let $S=S_{1} \times \cdots \times S_{r}$ be a direct product of nonabelian finite simple groups, and $\varphi$ an automorphism of $S$ transitively permuting the factors. Is it true that $E_{S, n}(\varphi)=S ?$

Here, recall, $E_{S, n}(\varphi)=\langle[x, \underbrace{\varphi, \ldots, \varphi}_{n}] \mid x \in S\rangle$, where commutators are taken in the semidirect product $S\langle\varphi\rangle$. In Lemma 3.9 we obtained an affirmative answer to Question 7.3 in the special case where the order of $\varphi$ is equal to the number of factors $r$, that is, when the stabilizer of a point in $\langle\varphi\rangle$ in the induced permutational action on $\left\{S_{1}, \ldots, S_{r}\right\}$ is trivial. However, in general Question 7.3 seems rather difficult and remains open. The first step would be to consider the case of $\varphi$ of prime order, when of course the open question is about a single nonabelian finite simple group and its automorphism of prime order. A significant headway in this direction was recently made by Robert Guralnick (private communication).

The reduction of Conjectures 7.1 and 7.2 to an affirmative answer to Question 7.3 can be conducted simultaneously based on the following proposition.

Proposition 7.4. Let $\alpha$ be an automorphism of a finite group $G$ such that $G=[G, \alpha]$. Assume that Question 7.3 has an affirmative answer. Then $E_{G, n}(\alpha)=G$.

Proof. Let $E=E_{G, n}(\alpha)$ for brevity. Let $G$ be a counterexample of minimal order, and let $M$ be a minimal $\alpha$-invariant normal subgroup of $G$. Then $G=M E$ and $M \not E$.

We need to consider two cases: $M$ can be an elementary abelian $q$-group for a prime $q$, or a direct product of isomorphic non-abelian simple groups.

If $M$ is an elementary abelian $q$-group, then the proof proceeds in exactly the same fashion as in the proof of Proposition 2.2 in the soluble case. Then $E \cap M=1$ by minimality of $M$ because $M$ is abelian and $G=M E$, and so on.

Thus, let $M=S_{1} \times \cdots \times S_{r}$, where the $S_{i}$ are isomorphic non-abelian simple groups. Suppose that $M \neq C_{M}(\alpha)$. By the affirmative answer to Question 7.3 every product over an orbit of $\alpha$ in the permutational action on $\left\{S_{1}, \ldots, S_{r}\right\}$ is contained in $E$ whenever $\alpha$ acts on this product nontrivially. Let $T$ be the product of those $S_{i}$ that are contained in $E$. Then $T$ is normal in $E\langle\alpha\rangle$, since if $S_{i} \leqslant E$ and $x \in E\langle\alpha\rangle$, then $S_{i}^{x} \leqslant E$ and $S_{i}^{x}$ is again one of the $S_{u}$. Since $T$ is also normal in $M$, we obtain that $T$ is normal in $M E\langle\alpha\rangle=G\langle\alpha\rangle$. By minimality of $M$ then $M=T \leqslant E$, a contradiction. Thus, $M=C_{M}(\alpha)$. Since $C_{G}(\alpha)$ normalizes $E$, then $[E, M] \leqslant M \cap E$ and $M \cap E$ is normal in $M$ and therefore in $M E\langle\alpha\rangle=G\langle\alpha\rangle$. Since $M \notin E$, by minimality of $M$ we have $M \cap E=1$ and therefore, $[E, M]=1$. As a result, $G=M \times E\langle\alpha\rangle$. This contradicts the hypothesis $G=[G, \alpha]$.

Theorem 7.5. Conjectures 7.1 and 7.2 are true if Question 7.3 has an affirmative answer.

Proof. As in the proofs of Theorems 1.1 and 1.2, consider the subnormal $g$-invariant subgroups

$$
[\ldots[[G, g], g], \ldots, g]
$$

and let $H$ be the smallest of these subgroups. Note that if $H=1$, then $g$ is a left-Engel element and therefore $g \in F(G)$ and there is nothing to prove. In any case, $H=[H, g]$.

Let $N=\left\langle H^{G}\right\rangle$ be the normal closure of $H$. By construction, the image of $g$ in $G / N$ is a left-Engel element and therefore belongs to the Fitting subgroup of $G / N$. If we prove that the generalized Fitting height of $N$ is at most $k$, then $N$, being a normal subgroup, 
is contained in $F_{k}^{*}(G)$ and then $g \in F_{k+1}^{*}(G)$, as required. Similarly, if we prove that the nonsoluble length of $N$ is at most $k$, then as a normal subgroup $N$ is contained in $R_{k}(G)$ and then $g \in R_{k}(G)$, as required. Since $H$ is subnormal in $G$, by Lemma 2.1(b) the generalized Fitting height and the nonsoluble length of its normal closure $N$ are the same as those of $H$. Therefore it suffices to estimate these parameters of $H$. But $H=[H, g] \leqslant E_{n}(g)$ by Proposition 7.4. Since $H$ is subnormal in $G$, the generalized Fitting height and the nonsoluble length of $H$ do not exceed the same parameters of $E_{n}(g)$. The result follows.

\section{REFERENCES}

[1] P. Hall and G. Higman, The $p$-length of a $p$-soluble group and reduction theorems for Burnside's problem, Proc. London Math. Soc. (3) 6 (1956), 1-42.

[2] B. Huppert Endliche Gruppen. I, Springer, Berlin, 1967.

[3] E. I. Khukhro and P. Shumyatsky, Words and pronilpotent subgroups in profinite groups, J. Austral. Math. Soc. 97, no. 3 (2014), 343-364.

[4] J. Wilson, On the structure of compact torsion groups, Monatsh. Math., 96 (1983), 57-66.

[5] E. I. Zelmanov, A solution of the Restricted Burnside Problem for groups of odd exponent, Izv. Akad. Nauk SSSR Ser. Mat., 54, 42-59; English transl., Math. USSR Izvestiya, 36 (1991), 41-60.

[6] E. I. Zelmanov, A solution of the Restricted Burnside Problem for 2-groups, Mat. Sbornik, 182, 568-592; English transl., Math. USSR Sbornik, 72 (1992), 543-565.

[7] E. I. Zelmanov, On periodic compact groups, Israel J. Math. 77, no. 1-2 (1992), 83-95.

Sobolev Institute of Mathematics, Novosibirsk, 630090, Russia, and

UNIVERSITY OF LINCOLN, U.K.

E-mail address: khukhro@yahoo.co.uk

Department of Mathematics, University of Brasilia, DF 70910-900, Brazil

E-mail address: pavel@unb.br 